\title{
Review Article \\ Review of Compounds and Pharmacological Effects of Delphinium
}

\author{
Sitan Chen, ${ }^{1}$ Lijun Meng, ${ }^{1}$ Fatma M. El-Demerdash, ${ }^{2,3}$ Li Zhou, ${ }^{1,2}$ Syed Arif Hussian Rizvi, ${ }^{2,4}$ \\ Lili Cui $\mathbb{D}^{1,5}$ and Wenyi Kang $\mathbb{D}^{1,2,5,6}$ \\ ${ }^{1}$ National $R$ \& D Center for Edible Fungus Processing Technology, Henan University, Kaifeng 475004, China \\ ${ }^{2}$ Joint International Research Laboratory of Food \& Medicine Resource Function, Henan University, Kaifeng 475004, \\ Henan Province, China \\ ${ }^{3}$ Department of Environmental Studies Institute of Graduate Studies and Research Alexandria University Alexandria, \\ Alexandria, Egypt \\ ${ }^{4}$ Institute of Plant and Environmental Protection (IPEP), National Agriculture Research Center (NARC) Islamabad, \\ Islamabad, Pakistan \\ ${ }^{5}$ Functional Food Engineering Technology Research Center, Kaifeng 475004, Henan Province, China \\ ${ }^{6}$ Kaifeng Key Laboratory of Functional Components in Health Food, Kaifeng 475004, Henan, China
}

Correspondence should be addressed to Lili Cui; cuill@vip.henu.edu.cn and Wenyi Kang; kangweny@hotmail.com

Received 15 June 2020; Revised 21 August 2020; Accepted 2 September 2020; Published 21 September 2020

Academic Editor: Pedro M. Mancini

Copyright (C) 2020 Sitan Chen et al. This is an open access article distributed under the Creative Commons Attribution License, which permits unrestricted use, distribution, and reproduction in any medium, provided the original work is properly cited.

Plants of Delphinium are herbal medicine used in the Tibet region with whole grass as a drug, which have the effects of analgesic, antibacterial, antipyretic, and anticancer. The main bioactive compounds are alkaloids, flavonoids, and sterols. This review summarized the compounds and pharmacological effects of Delphinium and provides a reference for further research on Delphinium.

\section{Introduction}

Delphinium of the Ranunculaceae family is widely distributed in the North temperate zone, with about 350 species worldwide. 173 species (150 endemic) of Delphinium are distributed in China [1]. Delphinium is composed of subgen. Delphinastrum, subgen. Delphinium, and subgen. Oligophyllon, in the world, of which subgen. Delphinastrum has the most species [2]. In China, there are 18 species of Delphinium used as folk medicine, which are used to treat bruises, rheumatism, toothache, and enteritis. In addition, four species of Delphinium can be used as soil pesticides for their effects of killing lice, mosquitoes, and fly larvae [3]. The main compounds of Delphinium are diterpenoid alkaloids, and most of them have physiological activities [4]. In addition, Delphinium also contains chemical constituents such as flavonoids and sterols. In recent years, with the development of analysis methods and increasing focus on
Delphinium, more and more chemical constituents and pharmacological activities of Delphinium had been researched. In this paper, the chemical constituents and pharmacological effects of Delphinium were reviewed in order to improve the development and utilization of the Delphinium resources.

\section{Chemical Constituents}

According to the research studies, the alkaloids are the main constituents with physiological activities in Delphinium and diterpenoid alkaloids are the most characteristic constituents with toxicity [5]. In addition, flavonoids and sterols are also present [6].

2.1. Diterpenoid Alkaloids. Diterpenoid alkaloids are derived from the amination of tetracycline diterpenoids or pentacyclic diterpenoids to heterocyclic systems containing 
$\beta$-aminoethanol, methylamine, or ethylamine nitrogen atoms [7]. There are abundant diterpenoid alkaloids in Delphinium, which can be classified into C-18 diterpenoid alkaloids, C-19 diterpenoid alkaloids, and C-20 diterpenoid alkaloids according to the carbon skeleton configuration [5]. Characteristic quaternary carbon signal and substituent signal are important information to distinguish different diterpene alkaloids.

C-18 diterpenoid alkaloids are the diterpenoid alkaloids whose $\mathrm{C}-18$ are mostly substituted by $\mathrm{C}(4)-\mathrm{H} / \mathrm{OH}$ or the ester group, and a few of them contain 3,4-epoxide. According to the oxygen-containing groups on C-7, they can be sorted into two types (Figure 1): lappacinitine-type and ranaconitine-type, and C-7 of the ranaconitine-type has an oxygen-substituent group. $\mathrm{C}_{4}\left(\delta_{\mathrm{c}} 30-40, \mathrm{~s}\right), \mathrm{C}_{8}\left(\delta_{\mathrm{c}} 73-84, \mathrm{~s}\right)$, and $\mathrm{C}_{11}\left(\delta_{\mathrm{c}} 47-55, \mathrm{~s}\right)$ are C-18's characteristic signals [8]. Characteristic signal of the ranaconitine-type on C-7 is at $\delta_{\mathrm{c}}$ 91-93 (s), and the characteristic signal of the lappacinitinetype on C-7 is at $\delta_{\mathrm{c}} 45-48(\mathrm{~s})$.

Most C-19 diterpene alkaloids are natural diterpenoid alkaloids and belong to pentacyclic diterpene alkaloids. According to the oxygen-containing groups on C-7 and the difference of skeleton, they can be classified into six types (Figure 2): lycoctonine-type, aconitine-type, 7,17-seco type $\left(\delta_{c 8} 137-139, s ; \delta_{c 10} 48-49, s ; \delta_{c 14} 216-218\right.$, s), lactone-type, pyro-type $\left(\delta_{c 8,15} 146-147, s\right)$, and rearranged-type $\left(\delta_{c 8}\right.$ $\left.40-55, \mathrm{~s} ; \delta_{\mathrm{c} 10} 44-45, \mathrm{~s} ; \delta_{\mathrm{c} 14} 210-211, \mathrm{~s}\right)$ [8]. The majority of C-19 diterpenoid alkaloids are lycoctonine-type and aconitine-type. The differences between them are that C-7 $\left(\delta_{\mathrm{c}}\right.$ 87-93) of the lycoctonine-type has an oxygen-substituent group and others do not have [9]. And, C-19 diterpenoid alkaloids isolated from Delphinium are mostly lycoctoninetype [10]. C-19 diterpene alkaloids contain oxygen substituent $\left(\delta_{\mathrm{H}} 3.0-5.0 ; \delta_{\mathrm{c}} 70-90\right)$ and methoxy substituent $\left(\delta_{\mathrm{H}} 3.2-3.6, \mathrm{~s} ; \delta_{\mathrm{c}} 55-59, \mathrm{q}\right) . \mathrm{C}_{4}\left(\delta_{\mathrm{c}} 37-41, \mathrm{~s}\right), \mathrm{C}_{8}$, and $\mathrm{C}_{11}\left(\delta_{\mathrm{c}}\right.$ $47-51, s)$ are C-19 characteristic signals [8].

C-20 diterpenoid alkaloids are tetracyclic diterpenes with a carbon skeleton of 20 carbon atoms and have a transa-ring alkaloid that connects C-19 to C-20 in N-ethyl or $\mathrm{N}-\beta$-hydroxyl ethyl [11]. Compared with C-18 and C-19, C-20 diterpene alkaloid skeletons are complex, and most of them have exocyclic double bond structures. At present, 22 types of C-20 diterpenoid alkaloids were found [12]. The C-20 diterpenoid alkaloids isolated from Delphinium mainly belong to atisine-type, veatchine-type, hetisine-type, hetidine-type, denudatine-type, delnudine-type [13], and vakognavine-type (Figure 3$)[14,15] . \mathrm{C}_{4}\left(\delta_{\mathrm{c}} 30-40, \mathrm{~s}\right), \mathrm{C}_{8}\left(\delta_{\mathrm{c}}\right.$ $30-50, s), \mathrm{C}_{11}\left(\delta_{\mathrm{c}} 30-40, \mathrm{~s}\right), \mathrm{C}_{16}\left(\delta_{\mathrm{c}}-143, \mathrm{~s}\right)$, and $\mathrm{C}_{17}\left(\delta_{\mathrm{H}^{-}}-5\right.$, brs; $\left.\delta_{\mathrm{c}^{-}}-110, \mathrm{t}\right)$ are C-20's characteristic signals. $\mathrm{C}_{6}\left(\delta_{\mathrm{c}} 17-20\right.$, s), $C_{7}\left(\delta_{\mathrm{c}} 31-35, s\right), \mathrm{C}_{10}\left(\delta_{\mathrm{c}} 36-40, \mathrm{~s}\right), \mathrm{C}_{14}\left(\delta_{\mathrm{c}} 25-26, \mathrm{~s}\right)$, and $\mathrm{C}_{20}\left(\delta_{\mathrm{c}} 50-54, \mathrm{~s}\right)$ are characteristic signals of atisine-type, and $\mathrm{C}_{6}\left(\delta_{\mathrm{c}}-24, \mathrm{~s}\right), \mathrm{C}_{7}\left(\delta_{\mathrm{c}}-43, \mathrm{~s}\right), \mathrm{C}_{10}\left(\delta_{\mathrm{c}} 48-51, \mathrm{~s}\right), \mathrm{C}_{14}\left(\delta_{\mathrm{c}}\right.$ $36-39, s)$, and $\mathrm{C}_{20}\left(\delta_{\mathrm{c}}-69, \mathrm{~s}\right)$ are characteristic signals of hetidine-type [16].

At present, 155 alkaloids were isolated from Delphinium, and the details are shown in Tables 1-3 and Figures 4-6. Based on the references listed in Tables 1-3, it can be summarized that $D$. anthriscifolium varietas and $D$. elatum and its varietas have been further studied in chemistry.

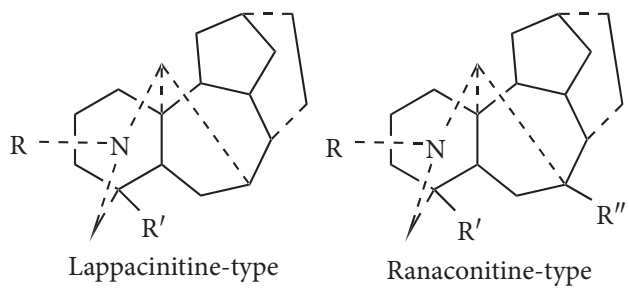

FIgURE 1: The skeleton structures of C-18 diterpene alkaloids.

2.2. Amide Alkaloids. The typical groups of amide alkaloids are acyl groups. 9 amide alkaloids were isolated from Delphinium, and the details are shown in Table 4 and Figure 7.

2.3. Other Alkaloids. Except diterpene alkaloids and amide alkaloids, one other alkaloid (No.155, anthriscifolsine A, $\mathrm{C}_{29} \mathrm{H}_{31} \mathrm{NO}_{7}$, [49]) was isolated from $D$. anthriscifolium var. majus. The structure is shown in Figure 8.

2.4. Other Compounds. Delphinium also contains compounds such as flavonoids and sterols. In recent years, other compounds isolated from Delphinium had also been reported, and a total of 13 nonalkaloids (Table 5 and Figure 9) were isolated from Delphinium.

\section{Biological Activities}

Plants of Delphinium are used with whole grass as medicine. According to the ancient Tibetan medicine Jingzhubencao, plants of Delphinium had analgesic, anti-inflammatory, and insecticidal effects [9]. Literature studies showed that plants of Delphinium have many pharmacological effects including antibacterial, antiepileptic, detoxification, and Alzheimer's disease treatment. In this section, this paper reviews the research studies on the antibacterial, analgesic, anti-inflammatory, antidepressant, and anticancer effects of Delphinium.

3.1. Antibacterial Activity. Hari et al. found that anthriscifoldine C $(5.0 \mathrm{mg} / \mathrm{mL})$ from D. brunonianum had a good inhibiting effect on Bacillus subtilis, Escherichia coli, and Salmonella flexnari, and its MIC were $24.0 \mu \mathrm{M}, 23.4 \mu \mathrm{M}$, and $24.2 \mu \mathrm{M}$, respectively, in vitro [61]. Ren et al. carried out the bacteriostatic test on the total alkaloids extracted from the roots of Delphinium and found that the MIC of the total alkaloids extracted on S. aureus and Aspergillus niger was $50 \mathrm{mg} / \mathrm{mL}$ in vitro [62].

3.2. Analgesic Activity. Zaheer et al. used the eddy current hot plate method and the tail flick reaction method to evaluate the analgesic activity of $D$. denudatum on Wistar albino rats. The experimental results showed that the response time of rats given $D$. denudatum ethanol extract and methanol fraction was longer than that of the propylene glycol group, and the effects of the high doses of the ethanol extract $(600 \mathrm{mg} / \mathrm{kg})$ and methanol fraction $(400 \mathrm{mg} / \mathrm{kg})$ were equal to that of the positive control group, indicating that 


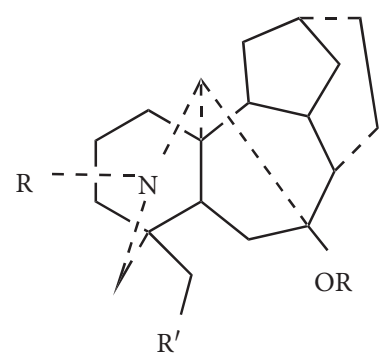

Lycoctonine-type

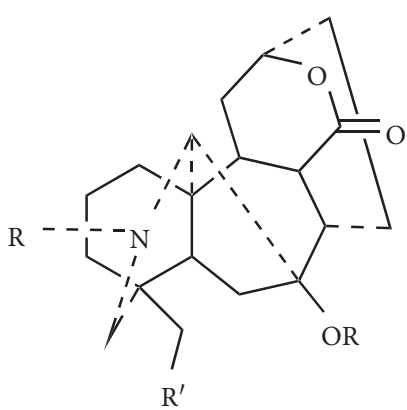

Lactone-type

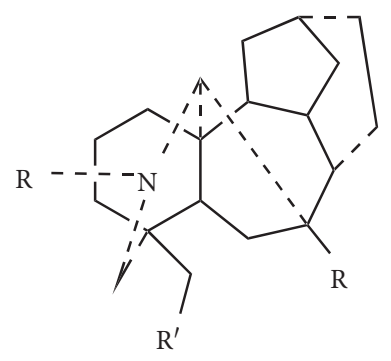

Aconitine-type

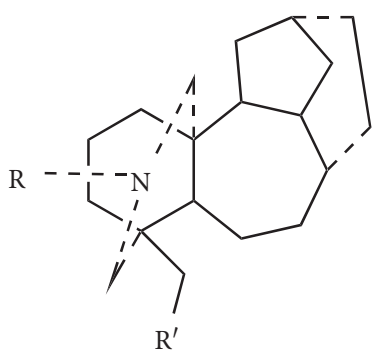

7,17-seco type

FIgURE 2: The skeleton structures of C-19 diterpene alkaloids.

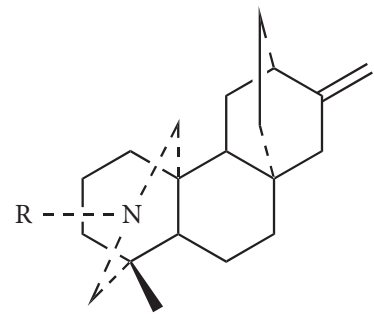

Atisine-type

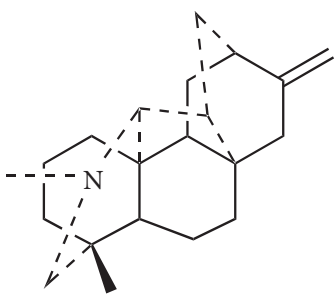

Hetidine-type

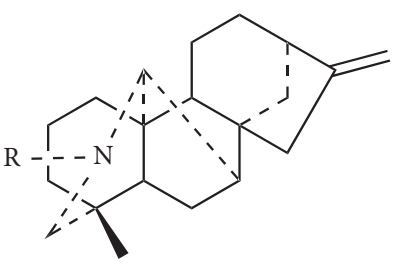

Veatchine-type

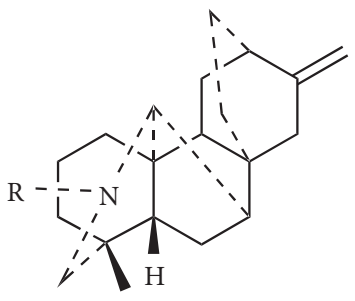

Denudatine-type

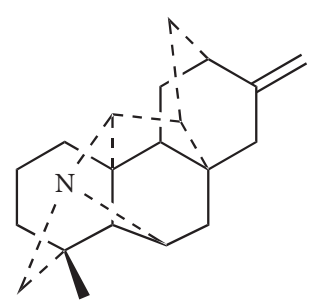

Hetisine-type

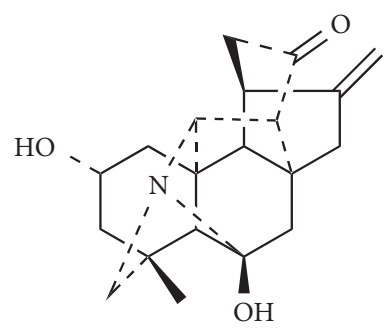

Delnudine-type

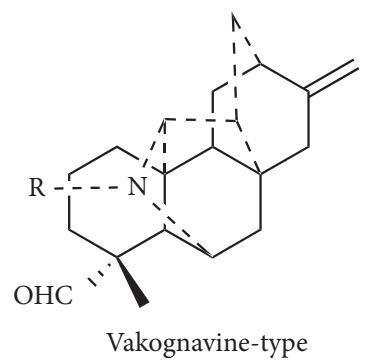

FIgURE 3: The skeleton structures of C-20 diterpene alkaloids.

D. denudatum had a good analgesic activity [63]. Nesterova et al. investigated chronic immune inflammation which was induced by injecting $0.1 \mathrm{~mL}$ of complete Freund's in outbred male rats, and the results showed that the $40 \%$ alcohol extract $(0.12 \mathrm{~mL} / \mathrm{kg})$ and total alkaloids $(0.05 \mathrm{mg} / \mathrm{kg})$ of Delphinium could significantly reduce the frequency of joint 
TABLe 1: C-18 diterpenoid alkaloids isolated from Delphinium.

\begin{tabular}{|c|c|c|c|c|c|}
\hline No. & Compound & Type & Source & Molecular formula & Reference \\
\hline 1 & Anthriscifolcine A & Ranaconitine & D. anthriscifolium var. savatieri & $\mathrm{C}_{26} \mathrm{H}_{38} \mathrm{NO}_{7}$ & [17] \\
\hline 2 & Anthriscifolcine B & Ranaconitine & D. anthriscifolium var. savatieri & $\mathrm{C}_{24} \mathrm{H}_{37} \mathrm{NO}_{6}$ & {$[17]$} \\
\hline 3 & Anthriscifolcine C & Ranaconitine & D. anthriscifolium var. savatieri & $\mathrm{C}_{25} \mathrm{H}_{37} \mathrm{NO}_{7}$ & {$[17]$} \\
\hline 4 & Anthriscifolcine D & Ranaconitine & D. anthriscifolium var. savatieri & $\mathrm{C}_{26} \mathrm{H}_{39} \mathrm{NO}_{7}$ & {$[17]$} \\
\hline 5 & Anthriscifolcine E & Ranaconitine & D. anthriscifolium var. savatieri & $\mathrm{C}_{24} \mathrm{H}_{37} \mathrm{NO}_{6}$ & {$[17]$} \\
\hline 6 & Anthriscifolcine $\mathrm{F}$ & Ranaconitine & D. anthriscifolium var. savatieri & $\mathrm{C}_{25} \mathrm{H}_{37} \mathrm{NO}_{8}$ & {$[18]$} \\
\hline 7 & Anthriscifolcine $G$ & Ranaconitine & D. anthriscifolium var. savatieri & $\mathrm{C}_{25} \mathrm{H}_{37} \mathrm{NO}_{7}$ & {$[18]$} \\
\hline 8 & Naviconine & Lappacinitine & D. naviculare var. lasiocarpum W. T. Wang. & $\mathrm{C}_{31} \mathrm{H}_{40} \mathrm{~N}_{2} \mathrm{O}_{9}$ & [19] \\
\hline 9 & Anthriscifolcone A & Ranaconitine & D. anthriscifolium var. majus & $\mathrm{C}_{27} \mathrm{H}_{39} \mathrm{NO}_{8}$ & {$[20]$} \\
\hline 10 & Anthriscifolcone B & Ranaconitine & D. anthriscifolium var. majus & $\mathrm{C}_{23} \mathrm{H}_{33} \mathrm{NO}_{7}$ & {$[20]$} \\
\hline 11 & Grandifline A & Ranaconitine & D. grandiflorum Linn & $\mathrm{C}_{22} \mathrm{H}_{33} \mathrm{NO}_{7}$ & {$[21]$} \\
\hline 12 & Tuguaconitine & Ranaconitine & D. grandiflorum & $\mathrm{C}_{23} \mathrm{H}_{35} \mathrm{NO}_{7}$ & {$[22]$} \\
\hline 13 & Linearilin & Ranaconitine & D. linearilobum (Trautv.) N. Busch & $\mathrm{C}_{24} \mathrm{H}_{39} \mathrm{NO}_{8}$ & [23] \\
\hline 14 & Anthriscifoltine A & Ranaconitine & D. anthriscifolium var. majus & $\mathrm{C}_{30} \mathrm{H}_{45} \mathrm{NO}_{9}$ & {$[24]$} \\
\hline 15 & Anthriscifoltine B & Ranaconitine & D. anthriscifolium var. majus & $\mathrm{C}_{28} \mathrm{H}_{43} \mathrm{NO}_{8}$ & {$[24]$} \\
\hline 16 & Anthriscifoltine C & Ranaconitine & D. anthriscifolium var. majus & $\mathrm{C}_{29} \mathrm{H}_{43} \mathrm{NO}_{9}$ & {$[25]$} \\
\hline 17 & Anthriscifoltine D & Ranaconitine & D. anthriscifolium var. majus & $\mathrm{C}_{32} \mathrm{H}_{41} \mathrm{NO}_{9}$ & {$[25]$} \\
\hline 18 & Anthriscifoltine E & Ranaconitine & D. anthriscifolium var. majus & $\mathrm{C}_{25} \mathrm{H}_{35} \mathrm{NO}_{8}$ & {$[25]$} \\
\hline 19 & Anthriscifoltine $\mathrm{F}$ & Ranaconitine & D. anthriscifolium var. majus & $\mathrm{C}_{23} \mathrm{H}_{33} \mathrm{NO}_{7}$ & {$[25]$} \\
\hline 20 & Anthriscifoltine G & Ranaconitine & D. anthriscifolium var. majus & $\mathrm{C}_{23} \mathrm{H}_{31} \mathrm{NO}_{7}$ & [25] \\
\hline
\end{tabular}

Table 2: C-19 diterpenoid alkaloids isolated from Delphinium.

\begin{tabular}{|c|c|c|c|c|c|}
\hline No. & Compound & Type & Source & $\begin{array}{l}\text { Molecular } \\
\text { formula }\end{array}$ & Reference \\
\hline 21 & Anthriscifoldine A & Lycoctonine & D. anthriscifolium var. savatieri & $\mathrm{C}_{25} \mathrm{H}_{37} \mathrm{NO}_{7}$ & {$[17]$} \\
\hline 22 & Anthriscifoldine B & Lycoctonine & D. anthriscifolium var. savatieri & $\mathrm{C}_{25} \mathrm{H}_{39} \mathrm{NO}_{7}$ & {$[17]$} \\
\hline 23 & Anthriscifoldine C & Lycoctonine & D. anthriscifolium var. savatieri & $\mathrm{C}_{27} \mathrm{H}_{41} \mathrm{NO}_{7}$ & [17] \\
\hline 24 & Naviculine & Lycoctonine & $\begin{array}{c}\text { D. naviculare var. lasiocarpum W. T. } \\
\text { Wang. }\end{array}$ & $\mathrm{C}_{26} \mathrm{H}_{42} \mathrm{NO}_{7}^{+}$ & {$[19]$} \\
\hline 25 & Naviconitine & Aconitine & $\begin{array}{c}\text { D. naviculare var. lasiocarpum W. T. } \\
\text { Wang. }\end{array}$ & $\mathrm{C}_{34} \mathrm{H}_{46} \mathrm{~N}_{2} \mathrm{O}_{9}$ & {$[19]$} \\
\hline 26 & Grandifline B & Lycoctonine & D. grandiflorum Linn & $\mathrm{C}_{25} \mathrm{H}_{39} \mathrm{NO}_{8}$ & {$[21]$} \\
\hline 27 & Grandifline C & Lycoctonine & D. grandiflorum Linn & $\mathrm{C}_{25} \mathrm{H}_{40} \mathrm{NO}_{7}^{+}$ & {$[21]$} \\
\hline 28 & Olivimine & Lycoctonine & D. grandiflorum & $\mathrm{C}_{24} \mathrm{H}_{37} \mathrm{NO}_{7}$ & {$[22]$} \\
\hline 29 & Hohenackeridine & Lycoctonine & D. grandiflorum & $\mathrm{C}_{22} \mathrm{H}_{31} \mathrm{NO}_{7}$ & {$[22]$} \\
\hline 30 & 14-O-Methyldelphinifoline & Lycoctonine & D. grandiflorum & $\mathrm{C}_{24} \mathrm{H}_{39} \mathrm{NO}_{7}$ & {$[22]$} \\
\hline 31 & N-Deethyldelphatine & Lycoctonine & D. grandiflorum & $\mathrm{C}_{24} \mathrm{H}_{39} \mathrm{NO}_{7}$ & {$[22]$} \\
\hline 32 & Browniine & Lycoctonine & D. grandiflorum & $\mathrm{C}_{25} \mathrm{H}_{41} \mathrm{NO}_{7}$ & {$[22]$} \\
\hline 33 & 14-Dehydrobrowniine & Lycoctonine & D. grandiflorum & $\mathrm{C}_{25} \mathrm{H}_{39} \mathrm{NO}_{7}$ & {$[22]$} \\
\hline 34 & Linearilobin & Aconitine & D. linearilobum (Trautv.) N. Busch & $\mathrm{C}_{37} \mathrm{H}_{46} \mathrm{~N}_{2} \mathrm{O}_{9}$ & {$[23]$} \\
\hline 35 & Melpheline & Lycoctonine & D. elatum & $\mathrm{C}_{24} \mathrm{H}_{37} \mathrm{NO}_{6}$ & {$[26]$} \\
\hline 36 & 19-Oxoisodelpheline & Lycoctonine & D. elatum & $\mathrm{C}_{25} \mathrm{H}_{37} \mathrm{NO}_{7}$ & {$[26]$} \\
\hline 37 & N-Deethyl-19-oxoisodelpheline & Lycoctonine & D. elatum & $\mathrm{C}_{23} \mathrm{H}_{33} \mathrm{NO}_{7}$ & {$[26]$} \\
\hline 38 & N-Deethyl-19-oxodelpheline & Lycoctonine & D. elatum & $\mathrm{C}_{23} \mathrm{H}_{33} \mathrm{NO}_{7}$ & {$[26]$} \\
\hline 39 & N-Formyl-4,19-secopacinine & Lycoctonine & D. elatum cv. Pacific Giant & $\mathrm{C}_{25} \mathrm{H}_{37} \mathrm{NO}_{7}$ & [27] \\
\hline 40 & Iminoisodelpheline & Lycoctonine & D. elatum cv. Pacific Giant & $\mathrm{C}_{23} \mathrm{H}_{33} \mathrm{NO}_{6}$ & {$[27]$} \\
\hline 41 & Iminodelpheline & Lycoctonine & D. elatum cv. Pacific Giant & $\mathrm{C}_{23} \mathrm{H}_{33} \mathrm{NO}_{6}$ & [27] \\
\hline 42 & Iminopaciline & Lycoctonine & D. elatum cv. Pacific Giant & $\mathrm{C}_{24} \mathrm{H}_{35} \mathrm{NO}_{6}$ & [27] \\
\hline 43 & 6-Dehydroeladine & Lycoctonine & D. elatum cv. Pacific Giant & $\mathrm{C}_{24} \mathrm{H}_{35} \mathrm{NO}_{6}$ & [27] \\
\hline 44 & Elapacidine & Lycoctonine & D. elatum cv. Pacific Giant & $\mathrm{C}_{24} \mathrm{H}_{37} \mathrm{NO}_{6}$ & {$[27]$} \\
\hline 45 & Yunnanensine A & $\begin{array}{l}\text { Rearranged- } \\
\text { type }\end{array}$ & D. yunnanense & $\mathrm{C}_{37} \mathrm{H}_{48} \mathrm{~N}_{2} \mathrm{O}_{9}$ & {$[28]$} \\
\hline 46 & Iliensine A & Lycoctonine & D. iliense & $\mathrm{C}_{40} \mathrm{H}_{55} \mathrm{NO}_{14}$ & {$[29]$} \\
\hline 47 & Iliensine B & Lycoctonine & D. iliense & $\mathrm{C}_{26} \mathrm{H}_{41} \mathrm{NO}_{8}$ & {$[29]$} \\
\hline 48 & Pseudophnine A & Lycoctonine & $\begin{array}{c}\text { D. pseudoaemulans C. Y. Yang et } \\
\text { B. Wang }\end{array}$ & $\mathrm{C}_{25} \mathrm{H}_{40} \mathrm{NO}_{7}^{+}$ & {$[30]$} \\
\hline 49 & Pseudophnine B & Lycoctonine & $\begin{array}{c}\text { D. pseudoaemulans C. Y. Yang et } \\
\text { B. Wang }\end{array}$ & $\mathrm{C}_{24} \mathrm{H}_{38} \mathrm{NO}_{7}^{+}$ & {$[30]$} \\
\hline
\end{tabular}


TABle 2: Continued.

\begin{tabular}{|c|c|c|c|c|c|}
\hline No. & Compound & Type & Source & $\begin{array}{l}\text { Molecular } \\
\text { formula }\end{array}$ & Reference \\
\hline 50 & Pseudophnine C & Lycoctonine & $\begin{array}{c}\text { D. pseudoaemulans C. Y. Yang et } \\
\text { B. Wang }\end{array}$ & $\mathrm{C}_{27} \mathrm{H}_{42} \mathrm{NO}_{7}^{+}$ & {$[30]$} \\
\hline 51 & Pseudophnine D & Lycoctonine & $\begin{array}{c}\text { D. pseudoaemulans C. Y. Yang et } \\
\text { B. Wang }\end{array}$ & $\mathrm{C}_{26} \mathrm{H}_{40} \mathrm{NO}_{7}^{+}$ & {$[30]$} \\
\hline 52 & Pseudorenines A & Lycoctonine & $\begin{array}{c}\text { D. pseudoaemulans C. Y. Yang et } \\
\text { B. Wang }\end{array}$ & $\mathrm{C}_{39} \mathrm{H}_{53} \mathrm{~N}_{2} \mathrm{O}_{11}^{+}$ & {$[30]$} \\
\hline 53 & Pseudorenines B & Lycoctonine & $\begin{array}{c}\text { D. pseudoaemulans C. Y. Yang et } \\
\text { B. Wang }\end{array}$ & $\mathrm{C}_{39} \mathrm{H}_{53} \mathrm{~N}_{2} \mathrm{O}_{11}^{+}$ & {$[30]$} \\
\hline 54 & Pseudonidine A & Lycoctonine & $\begin{array}{c}\text { D. pseudoaemulans C. Y. Yang et } \\
\text { B. Wang }\end{array}$ & $\mathrm{C}_{24} \mathrm{H}_{35} \mathrm{NO}_{7}$ & {$[30]$} \\
\hline 55 & Pseudonidine B & Lycoctonine & $\begin{array}{c}\text { D. pseudoaemulans C. Y. Yang et } \\
\text { B. Wang }\end{array}$ & $\mathrm{C}_{29} \mathrm{H}_{45} \mathrm{NO}_{8}$ & {$[30]$} \\
\hline 56 & Navicularine & Lycoctonine & D. naviculare var. lasiocarpum & $\mathrm{C}_{27} \mathrm{H}_{43} \mathrm{NO}_{8}$ & {$[31]$} \\
\hline 57 & Shawurensine & Lycoctonine & D. shawurense W. T. Wang & $\mathrm{C}_{37} \mathrm{H}_{52} \mathrm{~N}_{2} \mathrm{O}_{11}$ & {$[32]$} \\
\hline 58 & Sharwuphinine B & Lycoctonine & D. shawurense W. T. Wang & $\mathrm{C}_{26} \mathrm{H}_{40} \mathrm{NO}_{7}^{+}$ & {$[33]$} \\
\hline 59 & Ajacisine A & Lycoctonine & D. ajacis $\mathrm{L}$. & $\mathrm{C}_{31} \mathrm{H}_{44} \mathrm{~N}_{2} \mathrm{O}_{9}$ & {$[34]$} \\
\hline 60 & Ajacisine B & Lycoctonine & D. ajacis $\mathrm{L}$. & $\mathrm{C}_{32} \mathrm{H}_{46} \mathrm{~N}_{2} \mathrm{O}_{9}$ & {$[34]$} \\
\hline 61 & Ajacisine $\mathrm{C}$ & Lycoctonine & D. ajacis $\mathrm{L}$. & $\mathrm{C}_{31} \mathrm{H}_{42} \mathrm{~N}_{2} \mathrm{O}_{8}$ & {$[34]$} \\
\hline 62 & Ajacisine D & Lycoctonine & D. ajacis $\mathrm{L}$. & $\mathrm{C}_{30} \mathrm{H}_{42} \mathrm{~N}_{2} \mathrm{O}_{8}$ & {$[34]$} \\
\hline 63 & Ajacisine $\mathrm{E}$ & Lycoctonine & D. ajacis $\mathrm{L}$ & $\mathrm{C}_{30} \mathrm{H}_{42} \mathrm{~N}_{2} \mathrm{O}_{8}$ & {$[34]$} \\
\hline 64 & Caerudelphinine A & Lycoctonine & D. caeruleum Jacq.ex Camb & $\mathrm{C}_{25} \mathrm{H}_{39} \mathrm{NO}_{8}$ & {$[35]$} \\
\hline 65 & Grandiflodine B & Lycoctonine & D. grandiflorum & $\mathrm{C}_{33} \mathrm{H}_{48} \mathrm{~N}_{2} \mathrm{O}_{10}$ & {$[36]$} \\
\hline 66 & Majusine A & Lycoctonine & D. majus W. T. Wang & $\mathrm{C}_{32} \mathrm{H}_{44} \mathrm{~N}_{2} \mathrm{O}_{9}$ & {$[37]$} \\
\hline 67 & Majusine B & Lycoctonine & D. majus W. T. Wang & $\mathrm{C}_{24} \mathrm{H}_{37} \mathrm{NO}_{6}$ & {$[37]$} \\
\hline 68 & Majusine C & Lycoctonine & D. majus W. T. Wang & $\mathrm{C}_{26} \mathrm{H}_{37} \mathrm{NO}_{8}$ & [37] \\
\hline 69 & Davidisine A & Lycoctonine & D. davidii Franch. & $\mathrm{C}_{23} \mathrm{H}_{37} \mathrm{NO}_{7}$ & {$[38]$} \\
\hline 70 & Davidisine B & Lycoctonine & D. davidii Franch. & $\mathrm{C}_{24} \mathrm{H}_{37} \mathrm{NO}_{8}$ & {$[38]$} \\
\hline 71 & Laxicymine 1 & Lycoctonine & $\begin{array}{c}\text { D. laxicymosum var. pilostachyum W. T. } \\
\text { Wang }\end{array}$ & $\mathrm{C}_{24} \mathrm{H}_{35} \mathrm{NO}_{7}$ & [39] \\
\hline 72 & Laxicymisine 2 & Lycoctonine & $\begin{array}{c}\text { D. laxicymosum var. pilostachyum W. T. } \\
\text { Wang }\end{array}$ & $\mathrm{C}_{24} \mathrm{H}_{37} \mathrm{NO}_{7}$ & {$[39]$} \\
\hline 73 & Laxicyminine 3 & Lycoctonine & $\begin{array}{c}\text { D. laxicymosum var. pilostachyum W. T. } \\
\text { Wang }\end{array}$ & $\mathrm{C}_{24} \mathrm{H}_{35} \mathrm{NO}_{6}$ & {$[39]$} \\
\hline 74 & Tiantaishansine & Lycoctonine & $\begin{array}{c}\text { D. tiantaishanense W. J. Zhang et G. H. } \\
\text { Chen }\end{array}$ & $\mathrm{C}_{22} \mathrm{H}_{33} \mathrm{NO}_{7}$ & {$[40]$} \\
\hline 75 & Tiantaishannine & Lycoctonine & $\begin{array}{c}\text { D. tiantaishanense W. J. Zhang et G. H. } \\
\text { Chen }\end{array}$ & $\mathrm{C}_{26} \mathrm{H}_{39} \mathrm{NO}_{7}$ & {$[40]$} \\
\hline 76 & Tiantaishanmine & Lycoctonine & $\begin{array}{c}\text { D. tiantaishanense W. J. Zhang et G. H. } \\
\text { Chen }\end{array}$ & $\mathrm{C}_{25} \mathrm{H}_{35} \mathrm{NO}_{7}$ & {$[40]$} \\
\hline 77 & Trifoliolasine A & Lycoctonine & D. trifoliolatum Finet et Gagnep & $\mathrm{C}_{35} \mathrm{H}_{50} \mathrm{~N}_{2} \mathrm{O}_{9}$ & {$[41]$} \\
\hline 78 & Trifoliolasine B & Lycoctonine & D. trifoliolatum Finet et Gagnep & $\mathrm{C}_{36} \mathrm{H}_{52} \mathrm{~N}_{2} \mathrm{O}_{9}$ & {$[41]$} \\
\hline 79 & Trifoliolasine C & Lycoctonine & D. trifoliolatum Finet et Gagnep & $\mathrm{C}_{40} \mathrm{H}_{57} \mathrm{~N}_{3} \mathrm{O}_{11}$ & {$[41]$} \\
\hline 80 & $\begin{array}{l}\text { 14-Demethyl-14- } \\
\text { isobutyrylanhweidelphinine }\end{array}$ & Lycoctonine & D. pentagynum Lam. & $\mathrm{C}_{38} \mathrm{H}_{48} \mathrm{~N}_{2} \mathrm{O}_{11}$ & {$[42]$} \\
\hline 81 & $\begin{array}{l}\text { 14-Demethyl-14- } \\
\text { acetylanhweidelphinine }\end{array}$ & Lycoctonine & D. pentagynum Lam. & $\mathrm{C}_{36} \mathrm{H}_{44} \mathrm{~N}_{2} \mathrm{O}_{11}$ & {$[42]$} \\
\hline 82 & Giraldine G & Lycoctonine & D. giraldii & $\mathrm{C}_{40} \mathrm{H}_{57} \mathrm{~N}_{3} \mathrm{O}_{11}$ & {$[43]$} \\
\hline 83 & Giraldine $\mathrm{H}$ & Lycoctonine & D. giraldii & $\mathrm{C}_{41} \mathrm{H}_{59} \mathrm{~N}_{3} \mathrm{O}_{11}$ & {$[43]$} \\
\hline 84 & Giraldine I & Aconitine & D. giraldii & $\mathrm{C}_{22} \mathrm{H}_{35} \mathrm{NO}_{3}$ & {$[43]$} \\
\hline 85 & Giraldine D & Lycoctonine & D. giraldii & $\mathrm{C}_{24} \mathrm{H}_{37} \mathrm{NO}_{6}$ & {$[44]$} \\
\hline 86 & Giraldine E & Lycoctonine & D. giraldii & $\mathrm{C}_{25} \mathrm{H}_{39} \mathrm{NO}_{7}$ & {$[44]$} \\
\hline 87 & Giraldine F & Lycoctonine & D. giraldii & $\mathrm{C}_{23} \mathrm{H}_{33} \mathrm{NO}_{6}$ & {$[44]$} \\
\hline 88 & Campylocine & Lycoctonine & D. campylocentrum Maxim. & $\mathrm{C}_{25} \mathrm{H}_{37} \mathrm{NO}_{7}$ & {$[45]$} \\
\hline 89 & Campylotine & Lycoctonine & D. campylocentrum Maxim. & $\mathrm{C}_{24} \mathrm{H}_{37} \mathrm{NO}_{7}$ & {$[45]$} \\
\hline 90 & Davidiisine A & Lycoctonine & D. davidii Franch & $\mathrm{C}_{23} \mathrm{H}_{37} \mathrm{NO}_{7}$ & {$[46]$} \\
\hline 91 & Davidiisine B & Lycoctonine & D. davidii Franch & $\mathrm{C}_{24} \mathrm{H}_{37} \mathrm{NO}_{8}$ & {$[46]$} \\
\hline 92 & Ajadelphine & Lycoctonine & D. honanense var. piliteram W. T. Wang & $\mathrm{C}_{25} \mathrm{H}_{39} \mathrm{NO}_{7}$ & {$[47]$} \\
\hline 93 & Aconine & Aconitine & D. honanense var. piliteram W. T. Wang & $\mathrm{C}_{25} \mathrm{H}_{41} \mathrm{NO}_{9}$ & {$[47]$} \\
\hline 94 & Siwanine E & Lycoctonine & D. honanense var. piliteram W. T. Wang & $\mathrm{C}_{28} \mathrm{H}_{39} \mathrm{NO}_{9}$ & {$[47]$} \\
\hline
\end{tabular}


TABle 2: Continued.

\begin{tabular}{|c|c|c|c|c|c|}
\hline No. & Compound & Type & Source & $\begin{array}{l}\text { Molecular } \\
\text { formula }\end{array}$ & Reference \\
\hline 95 & Grandiflorine III & Aconitine & D. grandiflorum $\mathrm{L}$. & $\mathrm{C}_{26} \mathrm{H}_{39} \mathrm{NO}_{9}$ & {$[48]$} \\
\hline 96 & Isotalatizidine & Aconitine & D. grandiflorum L. & $\mathrm{C}_{23} \mathrm{H}_{37} \mathrm{NO}_{5}$ & {$[48]$} \\
\hline 97 & 14-O-Methyl isotalatizidine & Aconitine & D. grandiflorum L. & $\mathrm{C}_{24} \mathrm{H}_{39} \mathrm{NO}_{5}$ & {$[48]$} \\
\hline 98 & Anthranoyllycoctonine & Lycoctonine & D. grandiflorum $\mathrm{L}$. & $\mathrm{C}_{32} \mathrm{H}_{46} \mathrm{~N}_{2} \mathrm{O}_{8}$ & {$[48]$} \\
\hline 99 & Deoxylycoctonine & Lycoctonine & D. grandiflorum L. & $\mathrm{C}_{26} \mathrm{H}_{43} \mathrm{NO}_{6}$ & {$[48]$} \\
\hline 100 & Umbrosine & Lycoctonine & D. grandiflorum L. & $\mathrm{C}_{24} \mathrm{H}_{39} \mathrm{NO}_{6}$ & {$[48]$} \\
\hline 101 & Anthriscifolrine A & Lycoctonine & D. anthriscifolium var. majus & $\mathrm{C}_{25} \mathrm{H}_{37} \mathrm{NO}_{6}$ & [49] \\
\hline 102 & Anthriscifolrine B & Lycoctonine & D. anthriscifolium var. majus & $\mathrm{C}_{27} \mathrm{H}_{41} \mathrm{NO}_{8}$ & [49] \\
\hline 103 & Anthriscifolrine C & Lycoctonine & D. anthriscifolium var. majus & $\mathrm{C}_{27} \mathrm{H}_{41} \mathrm{NO}_{9}$ & [49] \\
\hline 104 & Anthriscifolrine D & Lycoctonine & D. anthriscifolium var. majus & $\mathrm{C}_{27} \mathrm{H}_{39} \mathrm{NO}_{9}$ & [49] \\
\hline 105 & Anthriscifolrine E & Lycoctonine & D. anthriscifolium var. majus & $\mathrm{C}_{26} \mathrm{H}_{39} \mathrm{NO}_{8}$ & [49] \\
\hline 106 & Anthriscifolrine $\mathrm{F}$ & Lycoctonine & D. anthriscifolium var. majus & $\mathrm{C}_{25} \mathrm{H}_{39} \mathrm{NO}_{7}$ & [49] \\
\hline 107 & Tianshanisine A & Lycoctonine & D. tianshanicum W. T. Wang & $\mathrm{C}_{30} \mathrm{H}_{41} \mathrm{NO}_{6}$ & {$[50]$} \\
\hline 108 & Tianshanisine B & Lycoctonine & D. tianshanicum W. T. Wang & $\mathrm{C}_{23} \mathrm{H}_{37} \mathrm{NO}_{5}$ & {$[50]$} \\
\hline 109 & Tianshanisine $\mathrm{C}$ & Lycoctonine & D. tianshanicum W. T. Wang & $\mathrm{C}_{25} \mathrm{H}_{39} \mathrm{NO}_{6}$ & {$[50]$} \\
\hline 110 & Tianshanisine D & Lycoctonine & D. tianshanicum W. T. Wang & $\mathrm{C}_{23} \mathrm{H}_{35} \mathrm{NO}_{5}$ & {$[50]$} \\
\hline 111 & Tianshanisine E & Lycoctonine & D. tianshanicum W. T. Wang & $\mathrm{C}_{23} \mathrm{H}_{35} \mathrm{NO}_{7}$ & {$[50]$} \\
\hline 112 & Elapacigine & Lycoctonine & Delphinium elatum cv. Pacific Giant & $\mathrm{C}_{23} \mathrm{H}_{31} \mathrm{NO}_{6}$ & {$[51]$} \\
\hline 113 & N-Deethyl-N-formylpaciline & Lycoctonine & Delphinium elatum cv. Pacific Giant & $\mathrm{C}_{25} \mathrm{H}_{37} \mathrm{NO}_{7}$ & {$[51]$} \\
\hline 114 & N-Deethyl-N-formylpacinine & Lycoctonine & Delphinium elatum cv. Pacific Giant & $\mathrm{C}_{24} \mathrm{H}_{33} \mathrm{NO}_{7}$ & [51] \\
\hline 115 & N-Formyl-4,19-secoyunnadelphinine & Lycoctonine & Delphinium elatum cv. Pacific Giant & $\mathrm{C}_{24} \mathrm{H}_{35} \mathrm{NO}_{7}$ & {$[51]$} \\
\hline
\end{tabular}

Table 3: C-20 diterpenoid alkaloids isolated from Delphinium.

\begin{tabular}{|c|c|c|c|c|c|}
\hline No. & Compound & Type & Source & Molecular formula & Reference \\
\hline 116 & Yunnanensine B & Hetisine & D. yunnanense & $\mathrm{C}_{28} \mathrm{H}_{37} \mathrm{NO}_{7}$ & {$[28]$} \\
\hline 117 & Yunnanensine C & Hetisine & D. yunnanense & $\mathrm{C}_{26} \mathrm{H}_{35} \mathrm{NO}_{6}$ & {$[28]$} \\
\hline 118 & Grandiflodine A & Hetisine & D. grandiflorum & $\mathrm{C}_{22} \mathrm{H}_{28} \mathrm{~N}_{2} \mathrm{O}_{3}$ & {$[36]$} \\
\hline 119 & Majusimine A & Vakognavine & D. majus W. T. Wang & $\mathrm{C}_{45} \mathrm{H}_{47} \mathrm{NO}_{15}$ & [37] \\
\hline 120 & Majusimine B & Vakognavine & D. majus W. T. Wang & $\mathrm{C}_{43} \mathrm{H}_{45} \mathrm{NO}_{14}$ & [37] \\
\hline 121 & Majusimine C & Vakognavine & D. majus W. T. Wang & $\mathrm{C}_{41} \mathrm{H}_{43} \mathrm{NO}_{13}$ & [37] \\
\hline 122 & Majusimine D & Vakognavine & D. majus W. T. Wang & $\mathrm{C}_{34} \mathrm{H}_{37} \mathrm{NO}_{12}$ & [37] \\
\hline 123 & Majusidine A & Hetisine & D. majus W. T. Wang & $\mathrm{C}_{22} \mathrm{H}_{29} \mathrm{NO}_{5}$ & [37] \\
\hline 124 & Majusidine B & Hetisine & D. majus W. T. Wang & $\mathrm{C}_{25} \mathrm{H}_{33} \mathrm{NO}_{4}$ & [37] \\
\hline 125 & Tiantaishandine & Hetisine & D. tiantaishanense W. J. Zhang et G. H. Chen & $\mathrm{C}_{29} \mathrm{H}_{33} \mathrm{NO}_{5}$ & {$[40]$} \\
\hline 126 & $\begin{array}{l}\text { 2-Dehydrodeacetylhetero } \\
\text { phylloidine }\end{array}$ & Hetidine & D. pentagynum Lam. & $\mathrm{C}_{21} \mathrm{H}_{25} \mathrm{NO}_{3}$ & {$[42]$} \\
\hline 127 & Davidiisine C & Hetidine & D. davidii Franch & $\mathrm{C}_{21} \mathrm{H}_{33} \mathrm{NO}_{4}$ & {$[46]$} \\
\hline 128 & 12-Epinapelline & Veatchine & D. honanense var. piliteram $\mathrm{W}$. T. Wang & $\mathrm{C}_{22} \mathrm{H}_{33} \mathrm{NO}_{3}$ & [47] \\
\hline 129 & Anthriscifolsine B & Hetisine & D. anthriscifolium var. majus & $\mathrm{C}_{24} \mathrm{H}_{31} \mathrm{NO}_{7}$ & {$[49]$} \\
\hline 130 & Anthriscifolsine C & Hetisine & D. anthriscifolium var. majus & $\mathrm{C}_{30} \mathrm{H}_{43} \mathrm{NO}_{7}$ & [49] \\
\hline 131 & Anthriscifolmine A & Denudatine & D. anthriscifolium var. savatieri & $\mathrm{C}_{25} \mathrm{H}_{37} \mathrm{NO}_{5}$ & {$[52]$} \\
\hline 132 & Anthriscifolmine B & Denudatine & D. anthriscifolium var. savatieri & $\mathrm{C}_{25} \mathrm{H}_{37} \mathrm{NO}_{6}$ & {$[52]$} \\
\hline 133 & Anthriscifolmine C & Hetisine & D. anthriscifolium var. savatieri & $\mathrm{C}_{29} \mathrm{H}_{31} \mathrm{NO}_{7}$ & {$[52]$} \\
\hline 134 & Trichodelphinines A & Hetisine & D. tichophorum Franch & $\mathrm{C}_{26} \mathrm{H}_{35} \mathrm{NO}_{5}$ & {$[53]$} \\
\hline 135 & Trichodelphinines B & Hetisine & D. tichophorum Franch & $\mathrm{C}_{24} \mathrm{H}_{33} \mathrm{NO}_{4}$ & {$[53]$} \\
\hline 136 & Trichodelphinines C & Hetisine & D. tichophorum Franch & $\mathrm{C}_{27} \mathrm{H}_{37} \mathrm{NO}_{5}$ & [53] \\
\hline 137 & Trichodelphinines D & Hetisine & D. tichophorum Franch & $\mathrm{C}_{24} \mathrm{H}_{31} \mathrm{NO}_{5}$ & {$[53]$} \\
\hline 138 & Trichodelphinines E & Hetisine & D. tichophorum Franch & $\mathrm{C}_{26} \mathrm{H}_{33} \mathrm{NO}_{5}$ & {$[53]$} \\
\hline 139 & Trichodelphinines F & Delnudine & D. tichophorum Franch & $\mathrm{C}_{28} \mathrm{H}_{33} \mathrm{NO}_{4}$ & {$[53]$} \\
\hline 140 & Flexiosine & Hetisine & D. flexuosum M. Bieb. & $\mathrm{C}_{36} \mathrm{H}_{43} \mathrm{NO}_{9}$ & {$[54]$} \\
\hline 141 & Tatsienenseine A & Vakognavine & D. tatsienense Franch & $\mathrm{C}_{43} \mathrm{H}_{45} \mathrm{NO}_{13}$ & {$[55]$} \\
\hline 142 & Tatsienenseine B & Hetisine & D. tatsienense Franch & $\mathrm{C}_{24} \mathrm{H}_{31} \mathrm{NO}_{4}$ & [55] \\
\hline 143 & Tatsienenseine $\mathrm{C}$ & Hetisine & D. tatsienense Franch & $\mathrm{C}_{24} \mathrm{H}_{31} \mathrm{NO}_{3}$ & {$[55]$} \\
\hline 144 & 13-(2-Methyl butyryl) azitine & Atisine & D. scabriflorum & $\mathrm{C}_{25} \mathrm{H}_{37} \mathrm{NO}_{3}$ & {$[56]$} \\
\hline 145 & Tatsienensine & Hetisine & D. tatsienense & $\mathrm{C}_{19} \mathrm{H}_{25} \mathrm{NO}_{2}$ & {$[57]$} \\
\hline
\end{tabular}




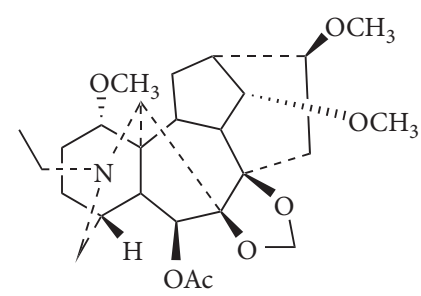

1 Anthriscifolcine A

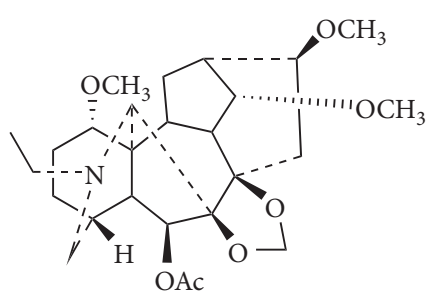

4 Anthriscifolcine D

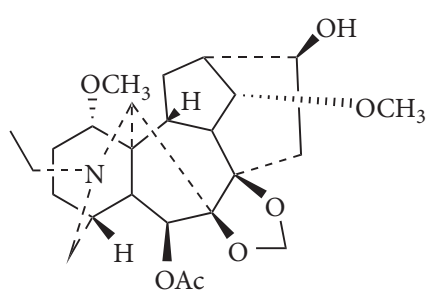

7 Anthriscifolcine G

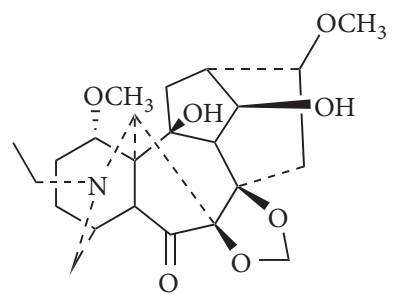

10 Anthriscifolcone B

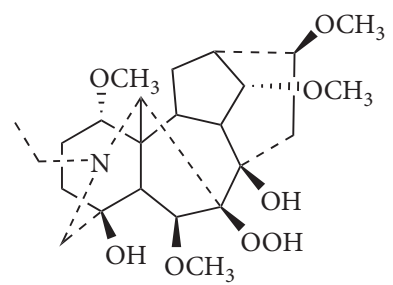

13 Linearilin

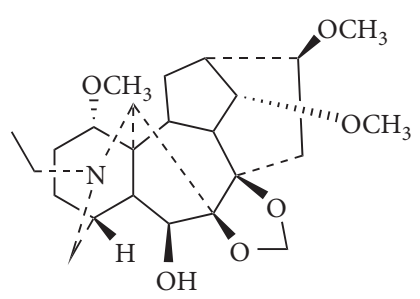

2 Anthriscifolcine B

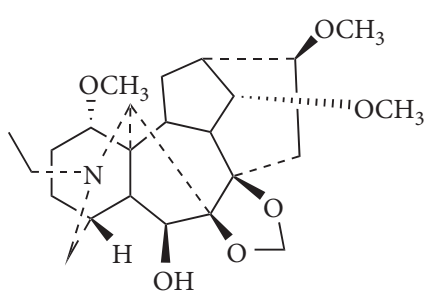

5 Anthriscifolcine E

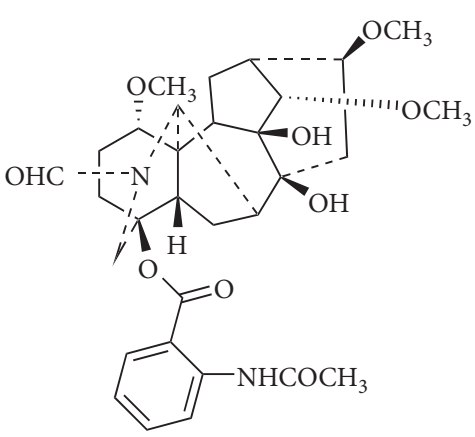

8 Naviconine

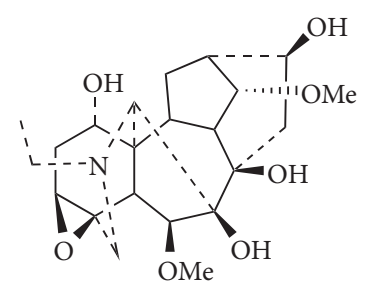

11 Grandifline A

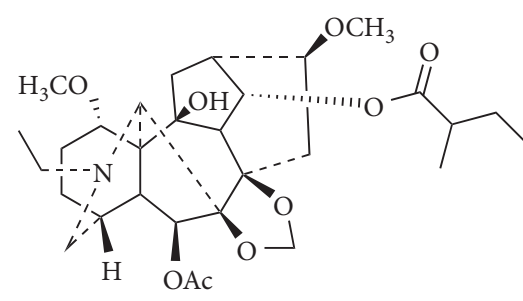

14 Anthriscifoltine A

FIgURE 4: Continued.

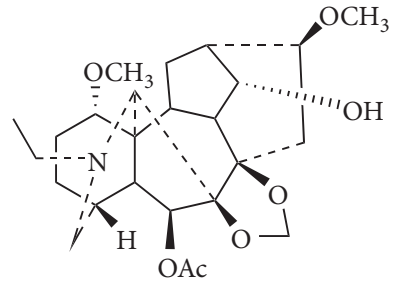

3 Anthriscifolcine C

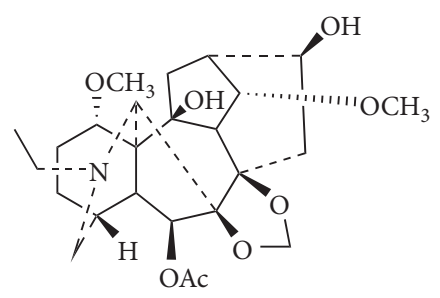

6 Anthriscifolcine F

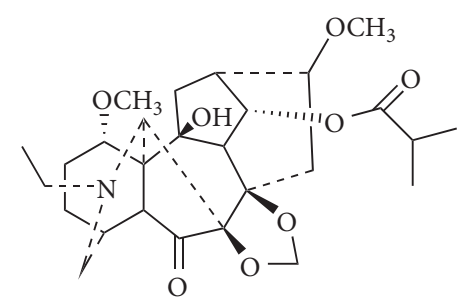

9 Anthriscifolcone A

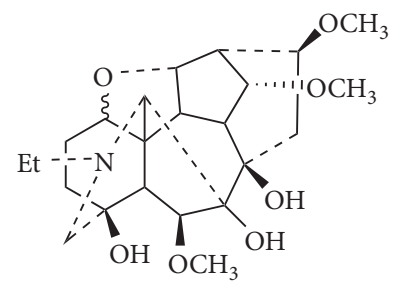

12 Tuguaconitine

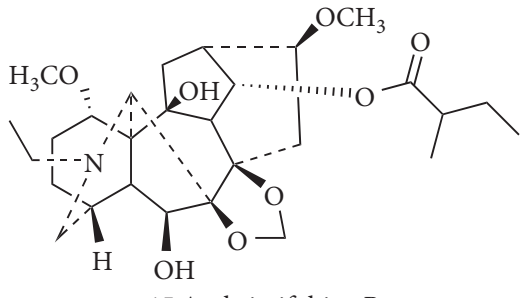

15 Anthriscifoltine B 

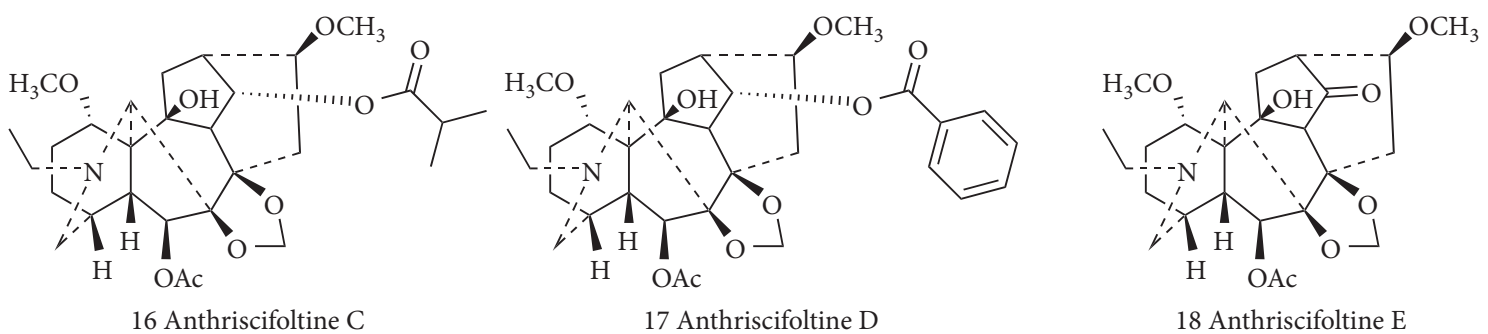

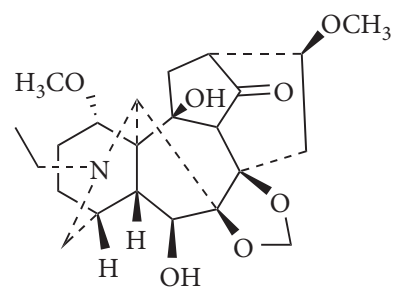

19 Anthriscifoltine F

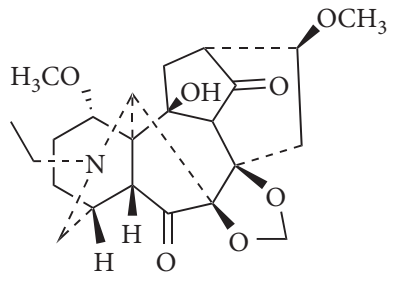

20 Anthriscifoltine $\mathrm{G}$

Figure 4: C-18 diterpenoid alkaloids isolated from Delphinium.

swelling in outbred male rats. On the 14th day, the rats in the total alkaloids $(0.05 \mathrm{mg} / \mathrm{kg})$ treatment group of Delphinium had no pain when their joints were bended, which indicated a good analgesic effect of Delphinium [64]. Through the hotplate method and the acetic acid writhing method, Suslov et al. found that the water extract $(0.5 \mathrm{~g} / \mathrm{kg})$ and the alcohol extract $(0.25 \mathrm{~g} / \mathrm{kg})$ of $D$. grandiflorum L. var. leiocarpum could prolong the pain threshold of mice, which was similar to the effect of acetaminophen $(0.2 \mathrm{~g} / \mathrm{kg})$, and performed a good analgesic effect [65].

3.3. Anti-Inflammatory Activity. Nesterova et al. found that the alkaloids and flavonoids in Delphinium had a good inhibitory effect on the inflammatory response through the experiment of the mice peritonitis model in the inflammatory exudation phase in vivo. Aqueous fraction of flavonoids $(25.0 \mathrm{mg} / \mathrm{kg})$ had a good therapeutic effect on the edema reaction caused by histamine $(0.1 \%)$, and alkaloids $(0.05 \mathrm{mg} / \mathrm{kg})$ showed a good anti-inflammatory effect on the inflammatory reaction caused by 5 -hydroxytryptamine $(0.5 \mathrm{mg} / \mathrm{kg})$ [66]. Andreeva and Liu established an acute inflammation model with increased capillary permeability induced by acetic acid in ICR male mice, and the results showed that the high- $(1.5 \mathrm{~g} / \mathrm{kg})$, medium- $(1.0 \mathrm{~g} / \mathrm{kg})$, and low-dose groups $(0.5 \mathrm{~g} / \mathrm{kg})$ of the total flavonoids extracted from $D$. grandiflorum with ethanol had good anti-inflammatory activity [67].

\subsection{Spiritual Influence}

3.4.1. Antidepressant Activity. Ebrahimzadeh et al. demonstrated that the extract $(250 \mathrm{mg} / \mathrm{kg}, 500 \mathrm{mg} / \mathrm{kg}$, and $1000 \mathrm{mg} / \mathrm{kg}$ ) of D. elbursense had good antidepressant activity by using the forced swimming experiment and the tail suspension experiment in mice. The results revealed that the extract at $1000 \mathrm{mg} / \mathrm{kg}$ had the same inhibitory activity as imipramine at $15 \mathrm{mg} / \mathrm{kg}$ in the control group [68].
3.4.2. Antianxiety Activity. Mohammad et al. found that the D. denudatum extract (200 and $400 \mathrm{mg} / \mathrm{kg}$ ) had a certain therapeutic effect on anxiety in Wistar albino rats and a better synergistic effect toward the Amaranthus spinosus extract $(100 \mathrm{mg} / \mathrm{kg})$ [69].

3.5. Anticancer Activity. Zheng et al. used the MTT method to determine the antihepatoma activity of the ethyl acetate extract from $D$. caeruleum in vitro. After giving $25,50,100$, and $200 \mu \mathrm{g} / \mathrm{mL}$ of HepG2 cells for 12, 24, and 48 hours, they found that the ethyl acetate extract from $D$. caeruleum had good antiliver cancer activity and had a good dose-effect and time-effect relationship on HepG2 cells and had less toxicity to L-02 cells. $\mathrm{IC}_{50}$ of the ethyl acetate extract from $D$. caeruleum on HepG2 cells was $28.8 \mu \mathrm{g} / \mathrm{mL}$ [70].

3.6. Antipulmonary Fibrosis Activity. In the study on pulmonary fibrosis induced by bleomycin in SD rats, Lin et al. found that after 14 days of gavage with $4 \mathrm{~g} / \mathrm{kg}, 2 \mathrm{~g} / \mathrm{kg}$, and $1 \mathrm{~g} / \mathrm{kg}$ extracts of $D$. trichophorum, the expression of collagen in the tissues during the pathological process of pulmonary fibrosis could be inhibited and the symptoms of pulmonary fibrosis in rats could be improved [71].

3.7. Antifeedant Activity. Shan et al. determined the antifood activity of 12 alkaloids isolated from $D$. naviculare var. lasiocarpum on Spodoptera exigua, and the results showed that the chemical shawurensine had a strong antifeedant activity with an $\mathrm{EC}_{50}$ of $0.45 \mathrm{mg} / \mathrm{cm}^{2}$ [31]. González and Guadaño extracted 5 alkaloids from Delphinium for activity determination against Spodoptera littoralis and Leptinotarsa decemlineata by choice feeding assays, and the results showed that cardiopetamine had the strongest activity against $S$. littoralis with the $\mathrm{EC}_{50}$ of $5.48 \mathrm{nmol} / \mathrm{cm}^{2}$ and 15 acetylcardiopetamine had the strongest activity against L. decemlineata with the $\mathrm{EC}_{50}$ of $12.86 \mathrm{nmol} / \mathrm{cm}^{2}$ [72] . 


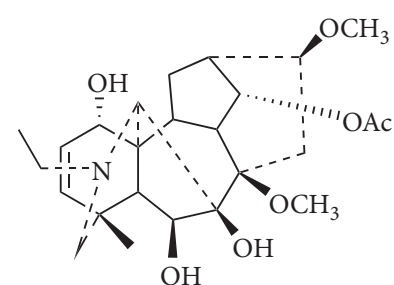

21 Anthriscifoldine A

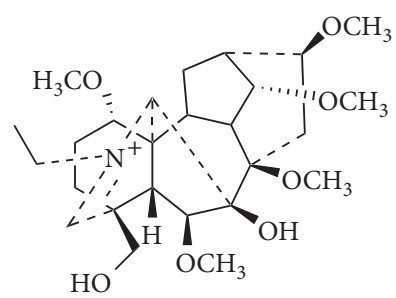

24 Naviculine

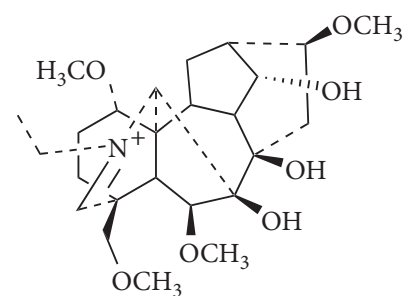

27 Grandifline C

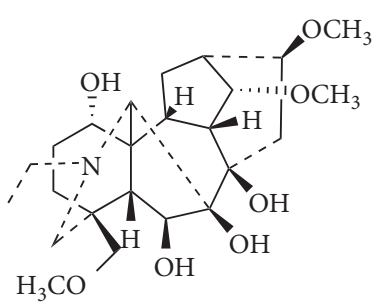

30 14-O-Methyldelphinifoline

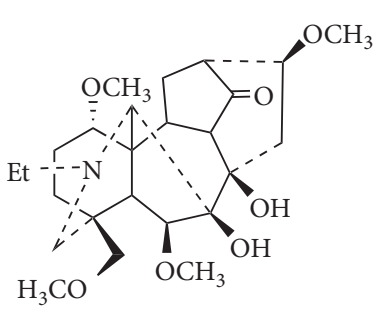

33 14-Dehydrobrowniine

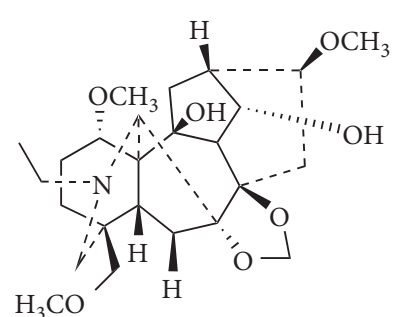

22 Anthriscifoldine B
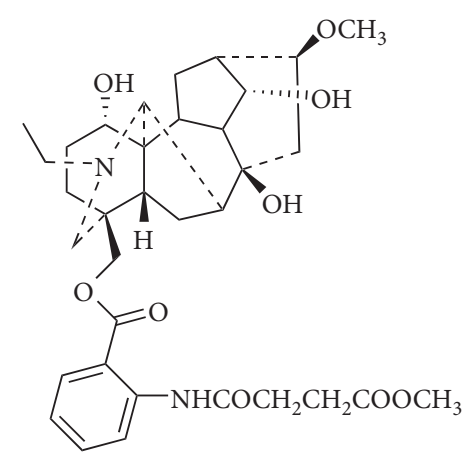

25 Naviconitine

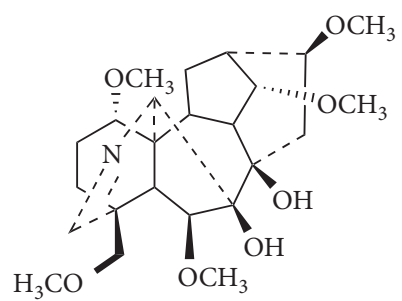

28 Olivimine

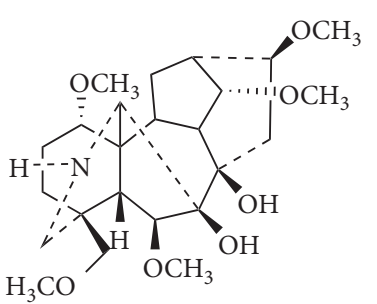

31 N-Deethyldelphatine

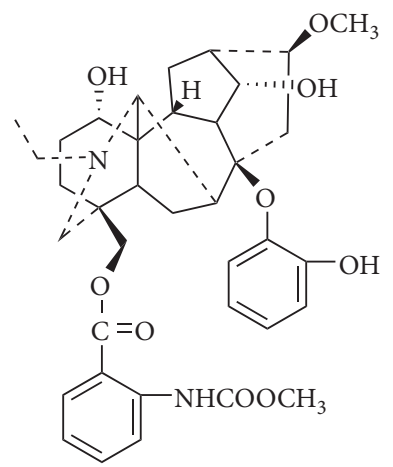

34 Linearilobin

FIGURE 5: Continued.

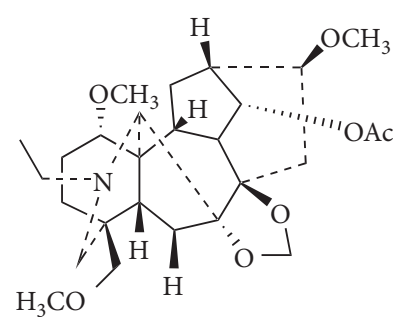

23 Anthriscifoldine C

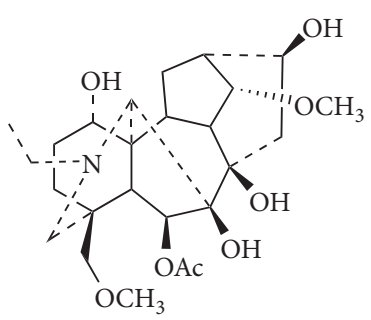

26 Grandifline B

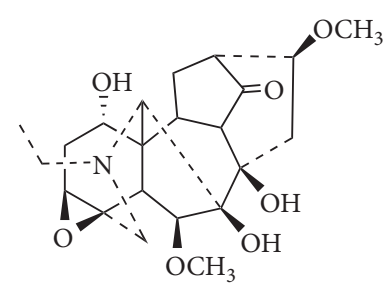

29 Hohenackeridine

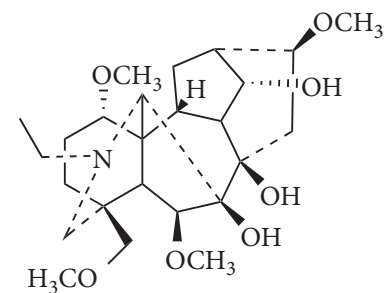

32 Browniine

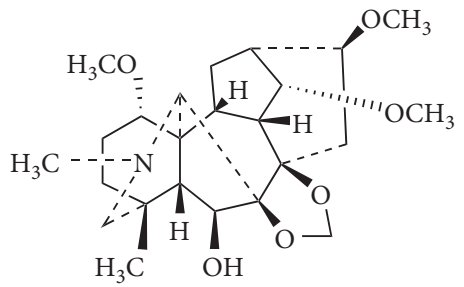

35 Melpheline 


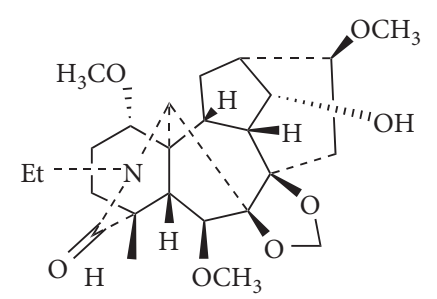

36 19-Oxoisodelpheline

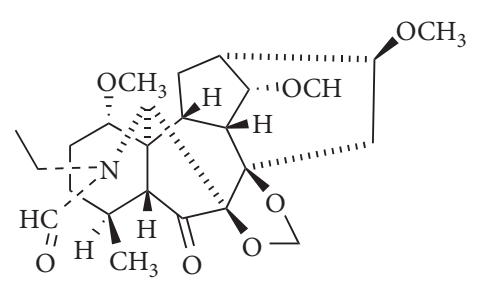

39 N-Formyl-4,19-secopacinine

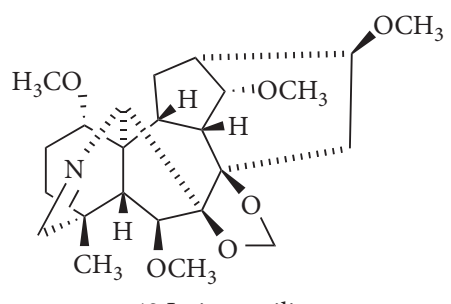

42 Iminopaciline

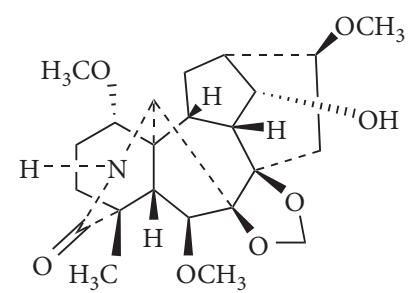

37 N-Deethyl-19-oxoisodelpheline

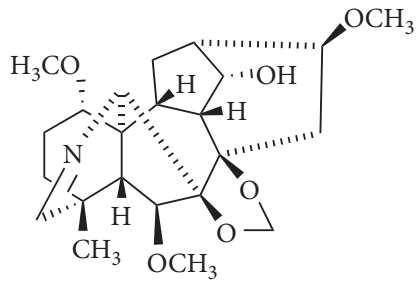

40 Iminoisodelpheline

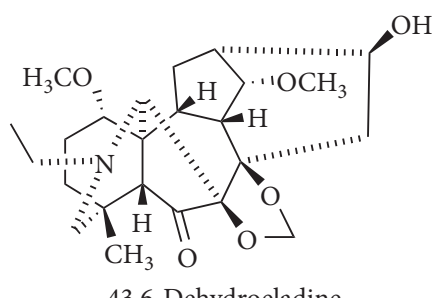

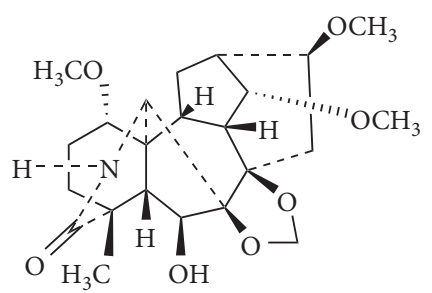

38 N-Deethyl-19-oxodelpheline

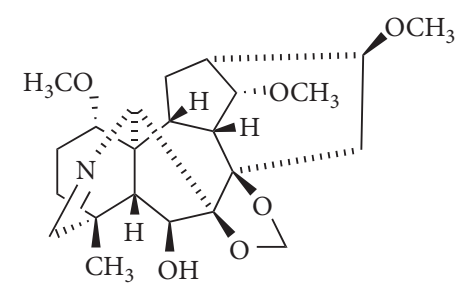

41 Iminodelpheline

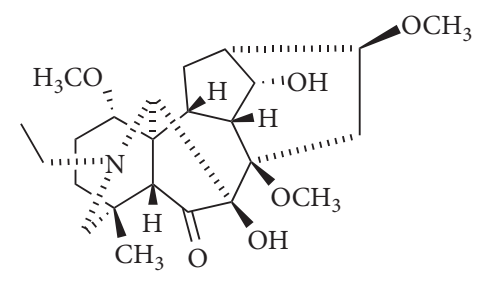

44 Elapacidine
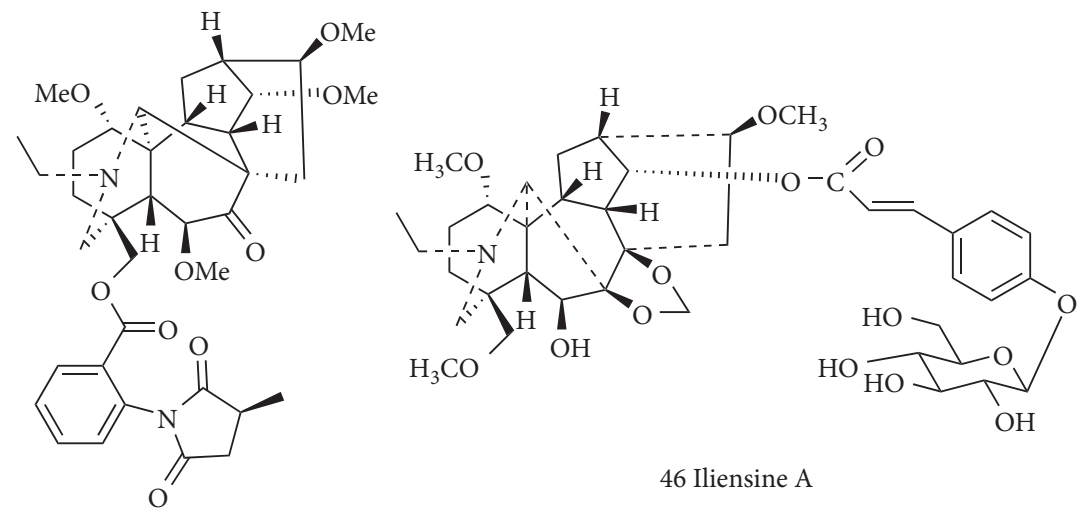

45 Yunnanensine A

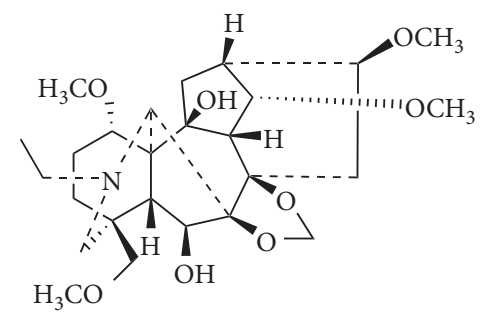

47 Iliensine B

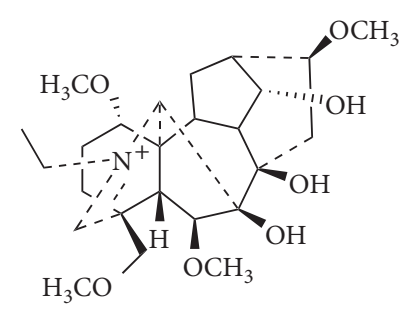

48 Pseudophnine A

Figure 5: Continued.

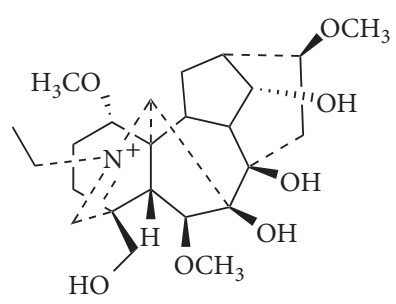

49 Pseudophnine B 


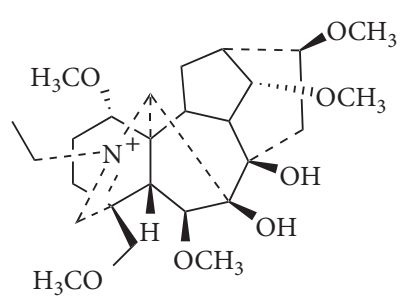

50 Pseudophnine C

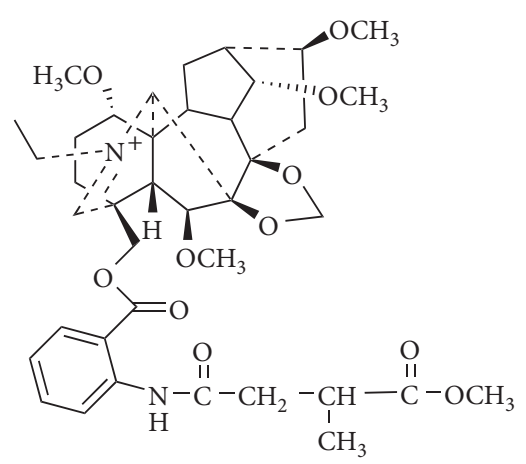

53 Pseudorenines B

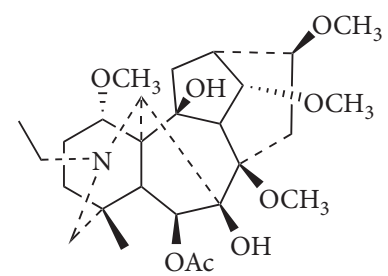

56 Navicularine
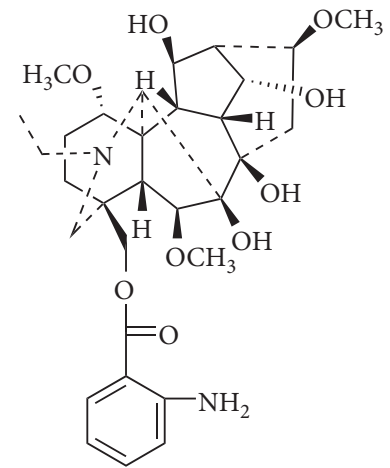

59 Ajacisine A

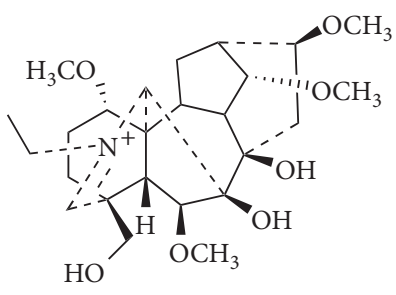

51 Pseudophnine D

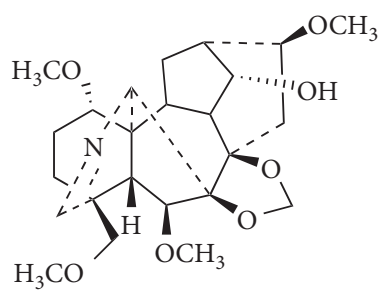

54 Pseudonidine A

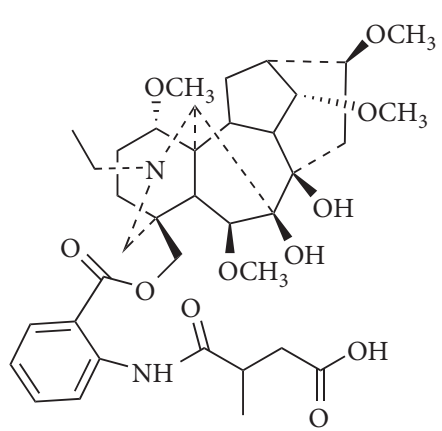

57 Shawurensine
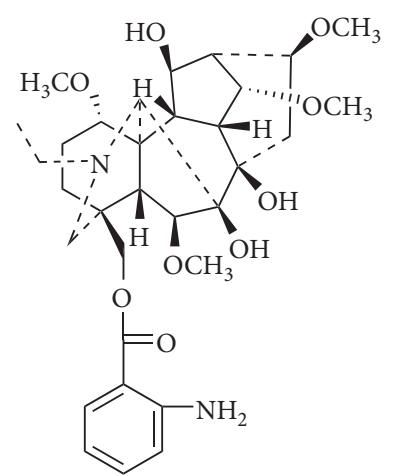

60 Ajacisine B

FIGURE 5: Continued.

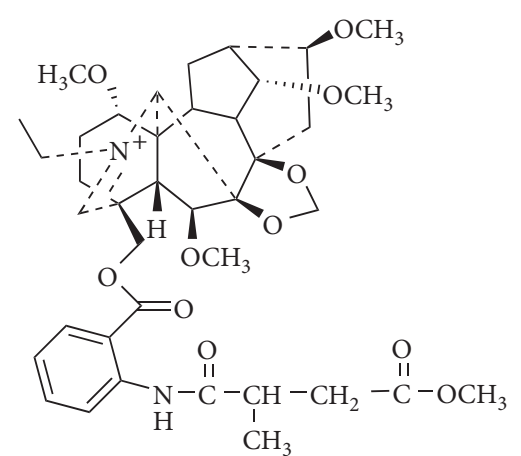

52 Pseudorenines A
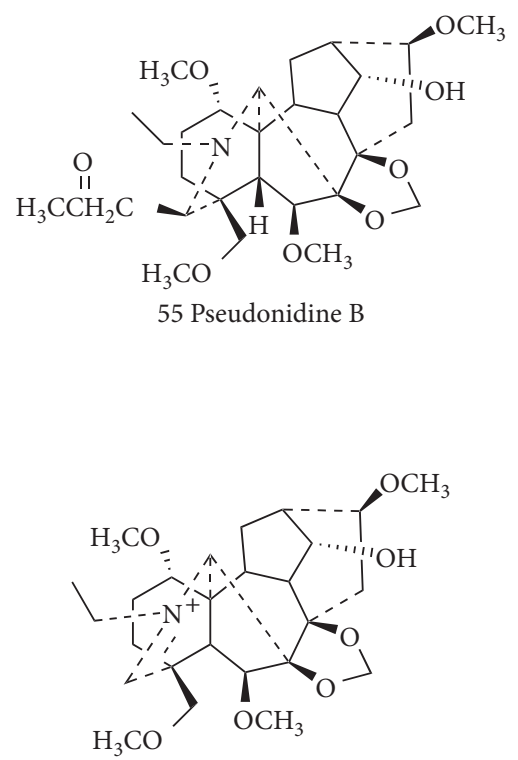

58 Sharwuphinine B
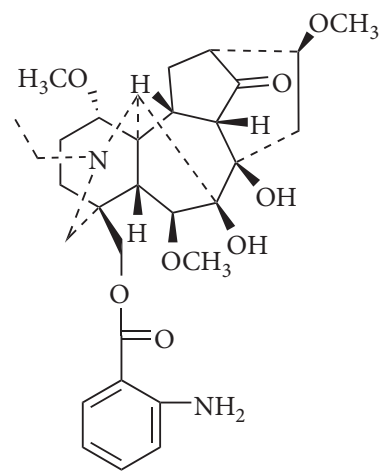

61 Ajacisine C 


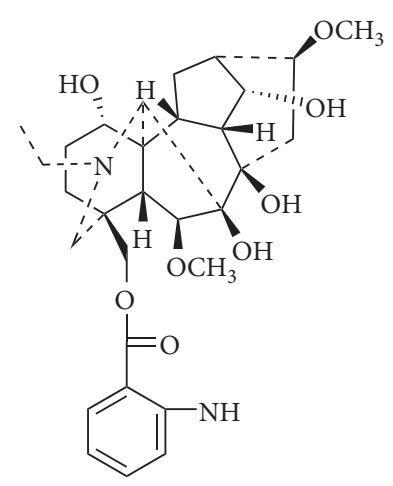

62 Ajacisine D

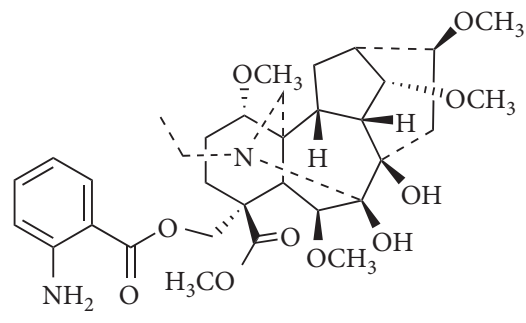

65 Grandiflodine B

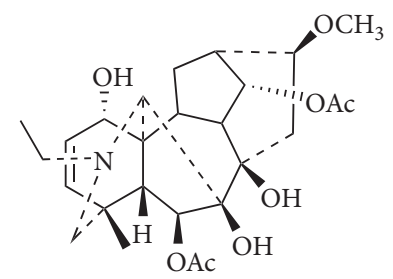

68 Majusine C

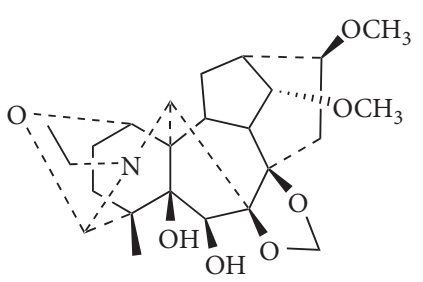

71 Laxicymine 1

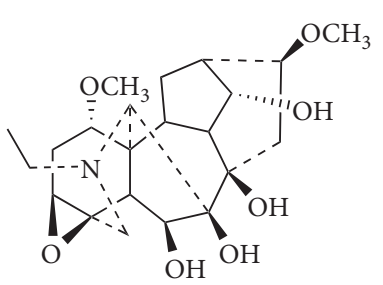

74 Tiantaishansine

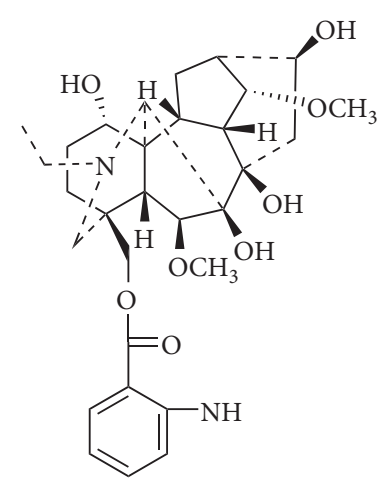

63 Ajacisine $\mathrm{E}$

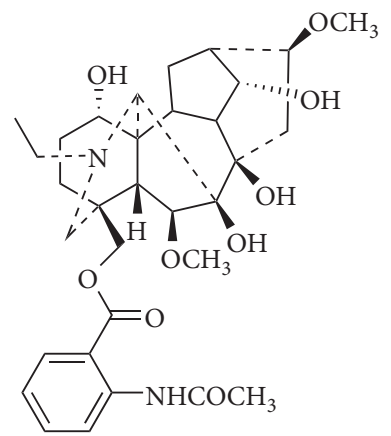

66 Majusine A

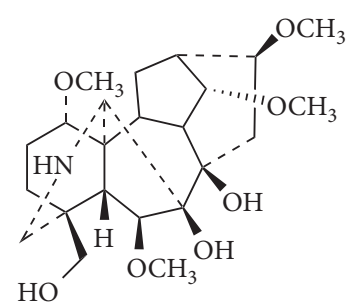

69 Davidisine A

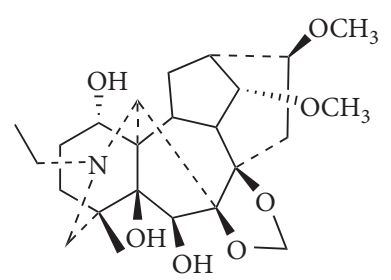

72 Laxicymisine 2

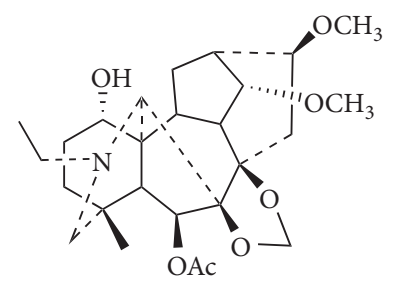

75 Tiantaishannine

Figure 5: Continued.

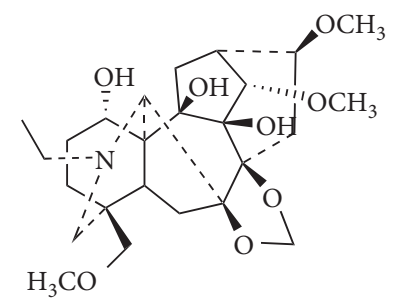

64 Caerudelphinine A

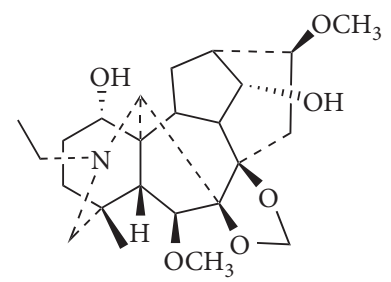

67 Majusine B

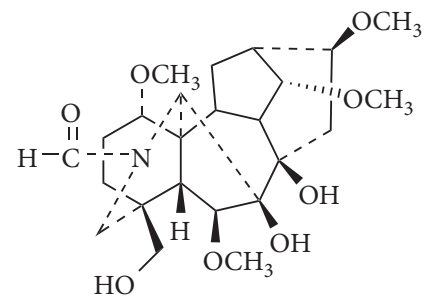

70 Davidisine B

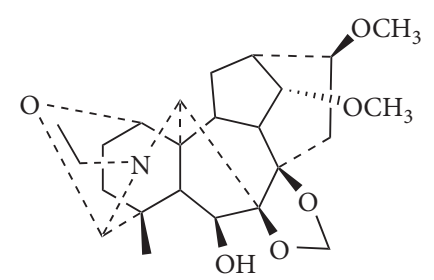

73 Laxicyminine 3

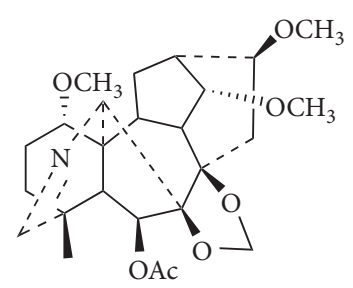

76 Tiantaishanmine 


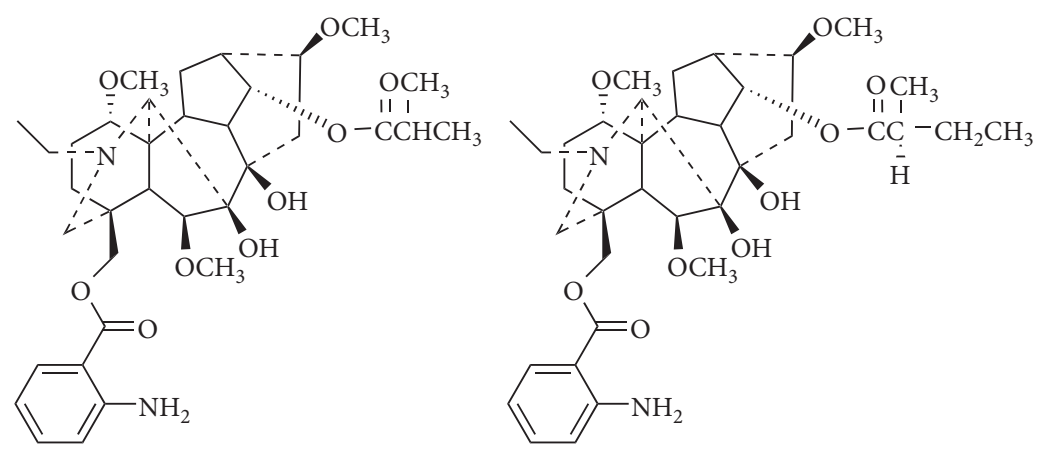

77 Trifoliolasine A

78 Trifoliolasine B

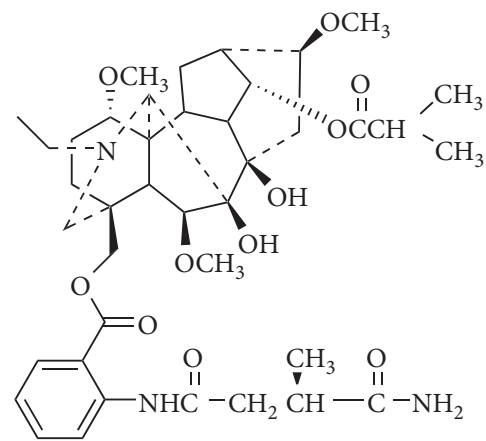

79 Trifoliolasine C

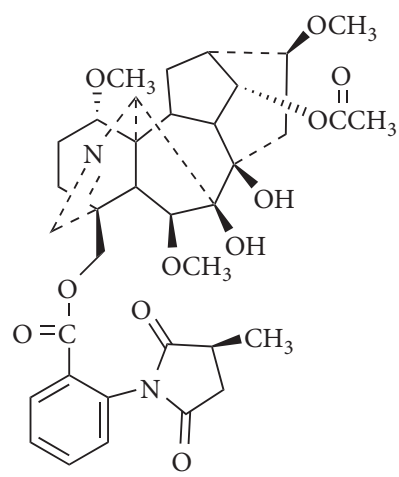

81 14-Demethyl-14-acetylanhweidelphinine

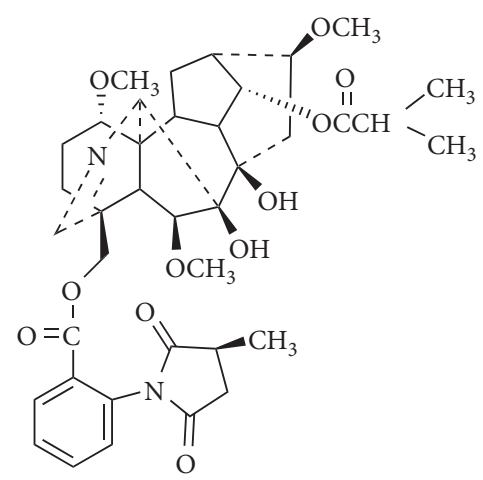

80 14-Demethyl-14isobutyrylanhweidelphinine

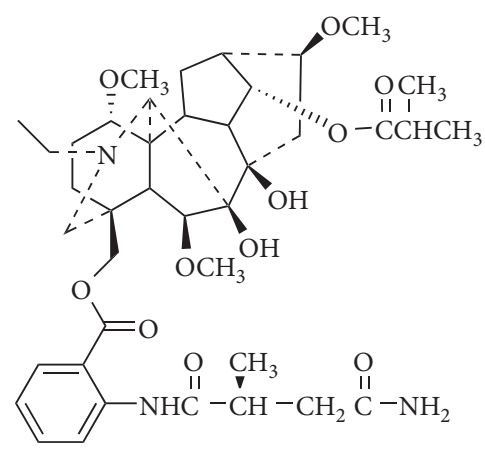

82 Giraldine G

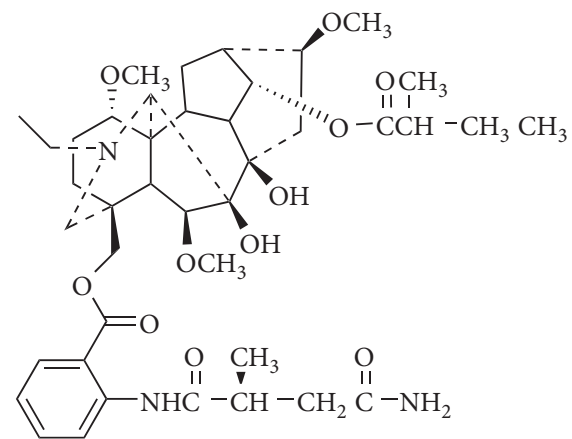

83 Giraldine $\mathrm{H}$

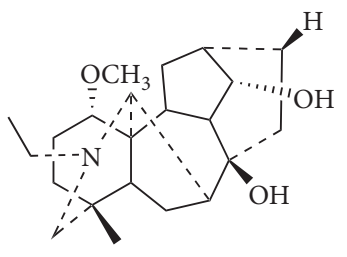

84 Giraldine I

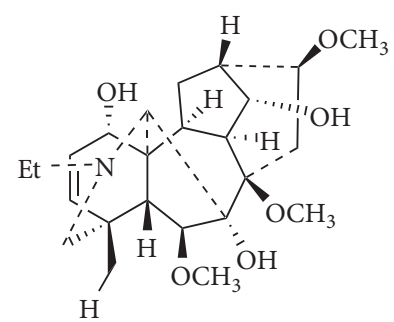

85 Giraldine D

Figure 5: Continued. 


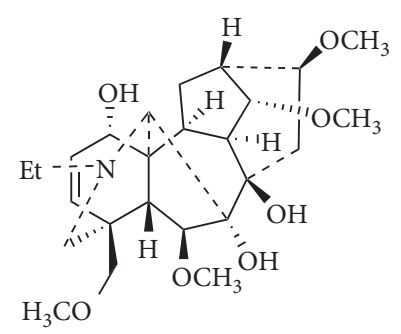

86 Giraldine E

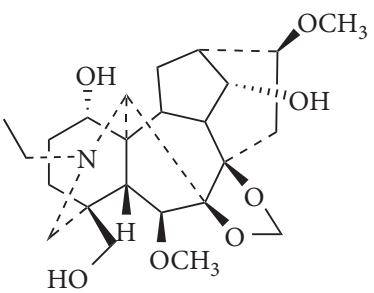

89 Campylotine

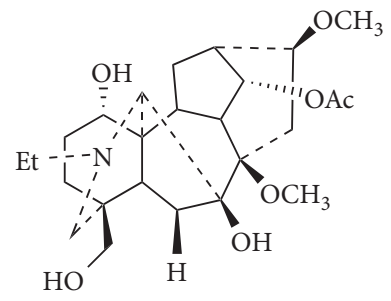

92 Ajadelphine

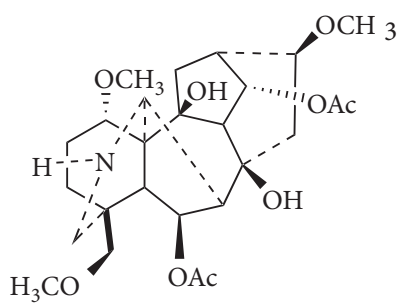

95 Grandiflorine III

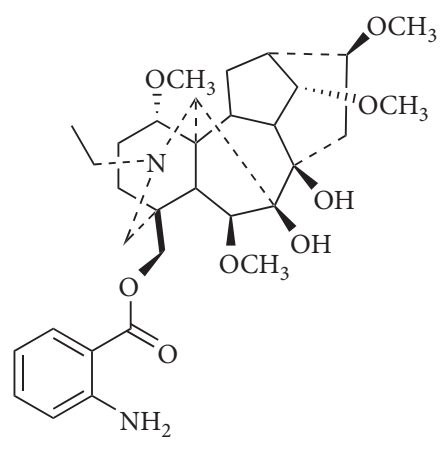

98 Anthranoyllycoctonine

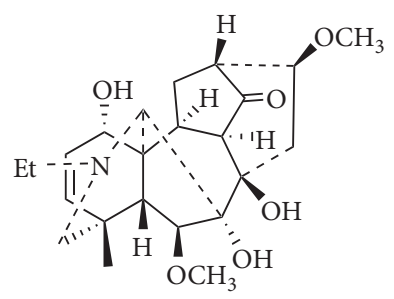

87 Giraldine F

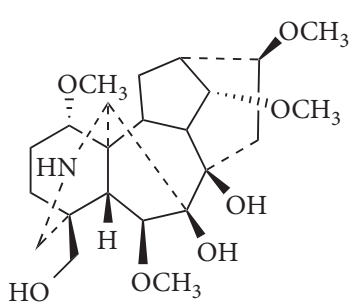

90 Davidiisine A

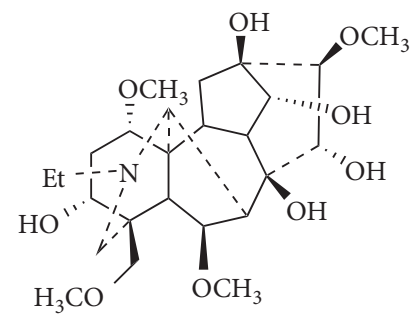

93 Aconine

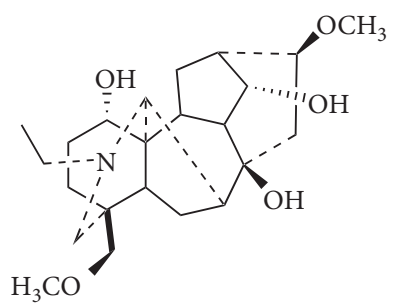

96 Isotalatizidine

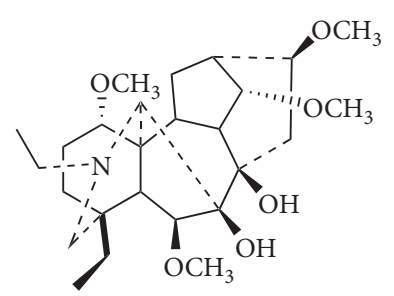

99 Deoxylycoctonine

FIgURE 5: Continued.

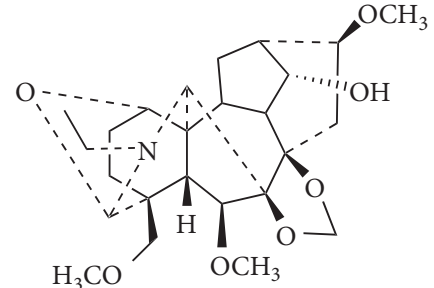

88 Campylocine

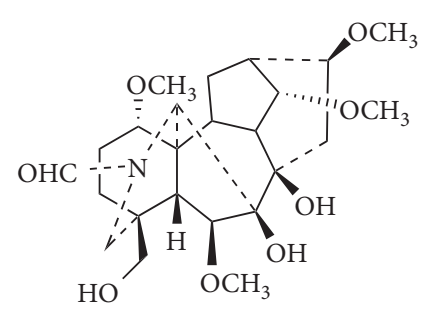

91 Davidiisine B

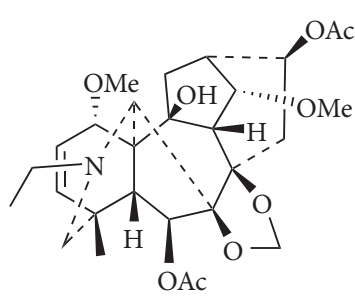

94 Siwanine E

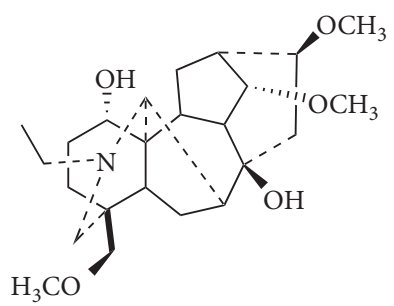

97 14-O-Methyl isotalatizidine

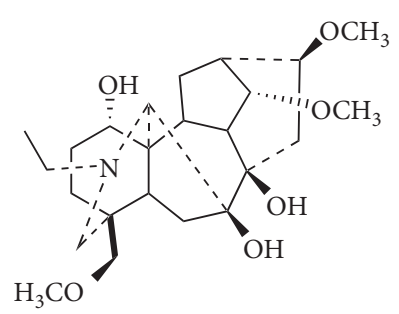

100 Umbrosine 


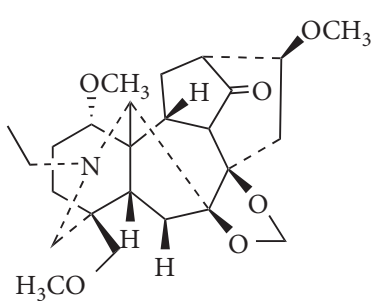

101 Anthriscifolrine A

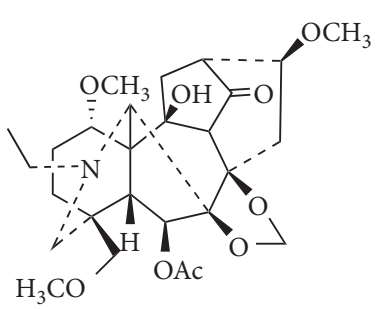

104 Anthriscifolrine D

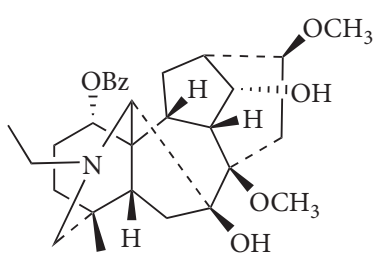

107 Tianshanisine A

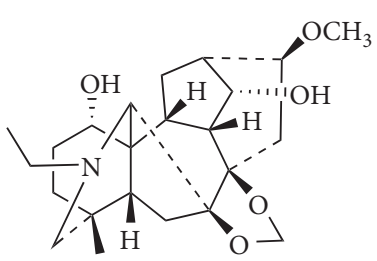

110 Tianshanisine D

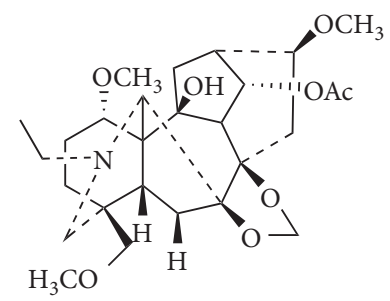

102 Anthriscifolrine B

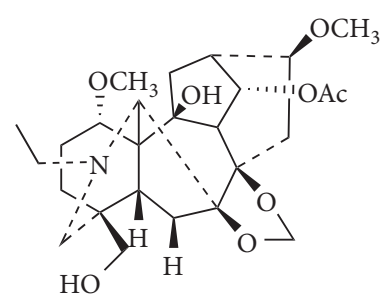

105 Anthriscifolrine E

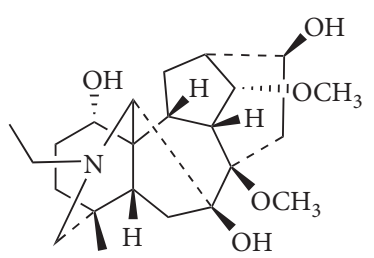

108 Tianshanisine B

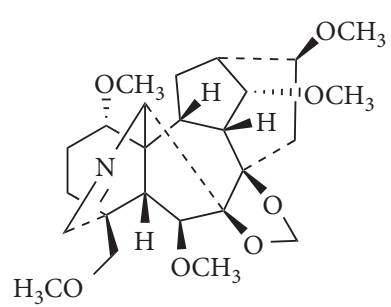

111 Tianshanisine E

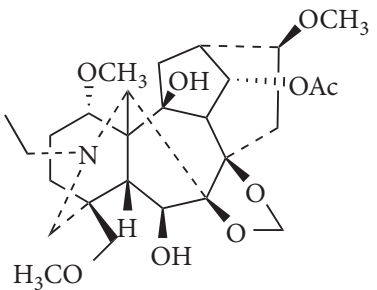

103 Anthriscifolrine C

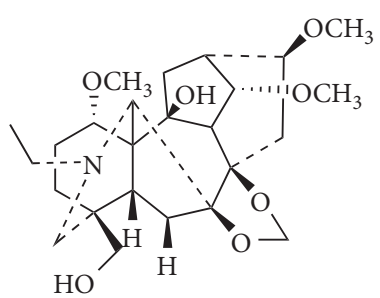

106 Anthriscifolrine F

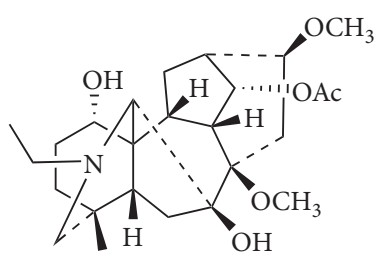

109 Tianshanisine C

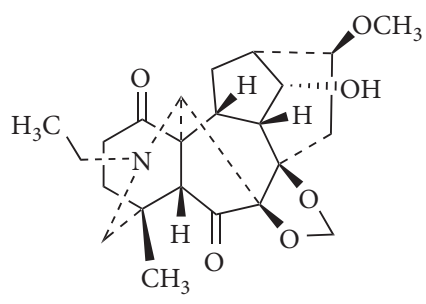

112 Elapacigine
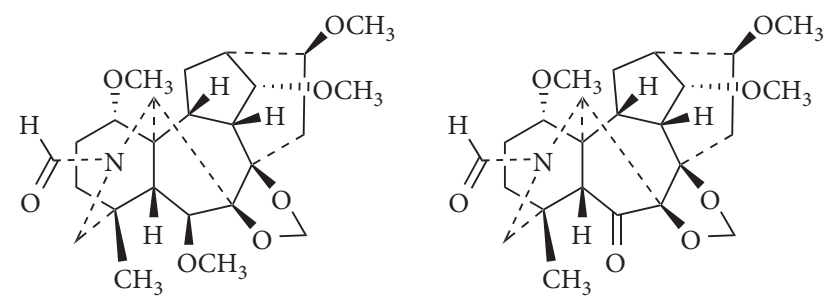

113 N-Deethyl-N-formylpaciline

114 N-Deethyl-N-formylpacinine

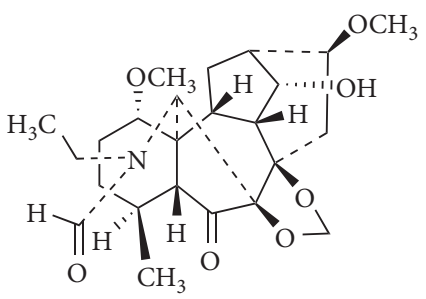

115 N-Formyl-4,19-secoyunnadelphinine

FIgURE 5: C-19 diterpenoid alkaloids isolated from Delphinium. 


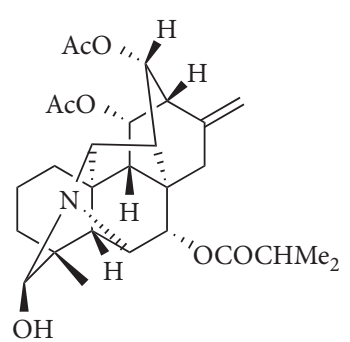

116 Yunnanensine B

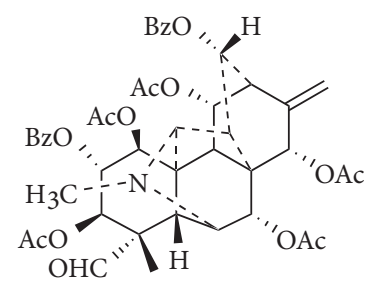

119 Majusimine A

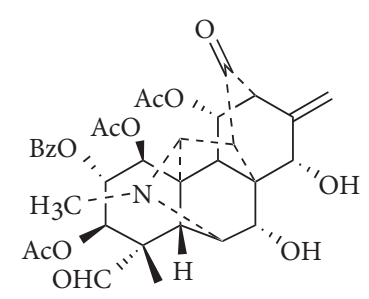

122 Majusimine D

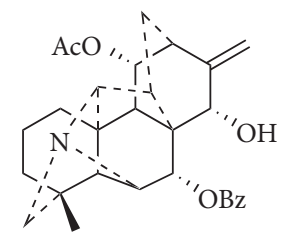

125 Tiantaishandine

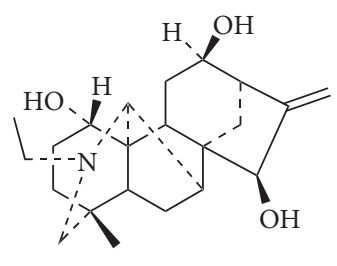

128 12-Epinapelline

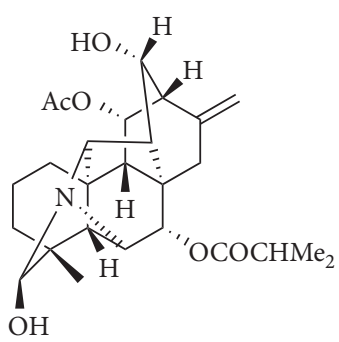

117 Yunnanensine C

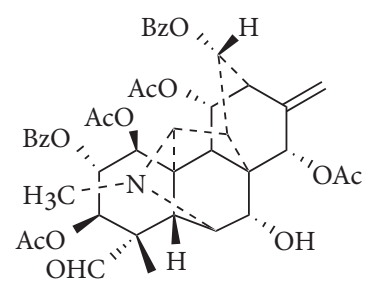

120 Majusimine B

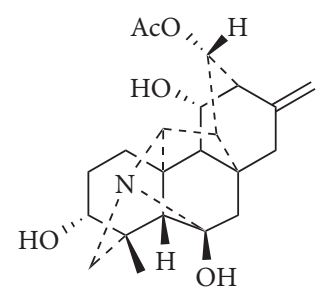

123 Majusidine A

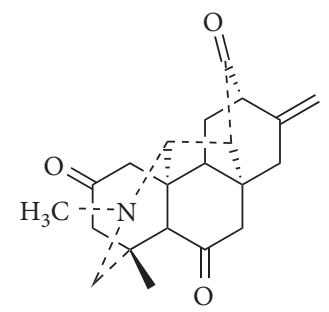

126 2-Dehydrodeacetylhetero phylloidine

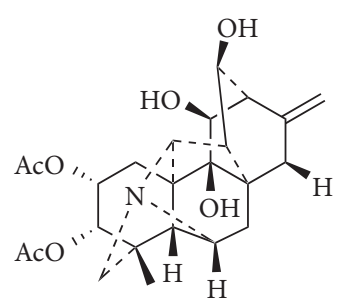

129 Anthriscifolsine B

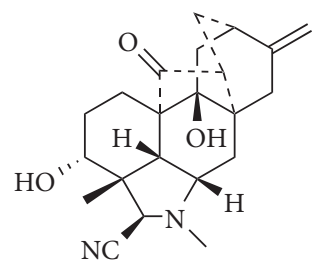

118 Grandiflodine A

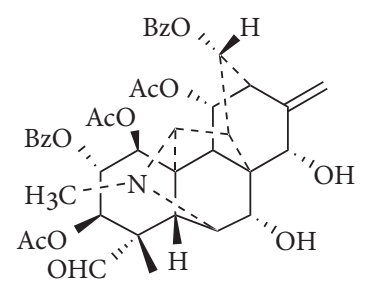

121 Majusimine C

$\mathrm{H}_{3} \mathrm{C}$

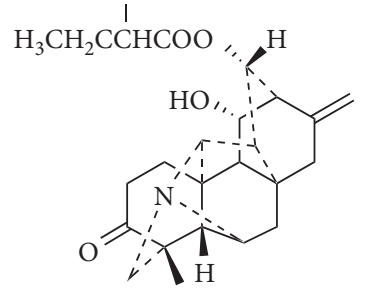

124 Majusidine B

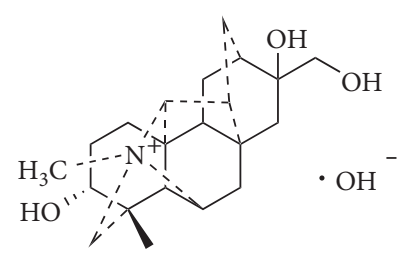

127 Davidiisine C

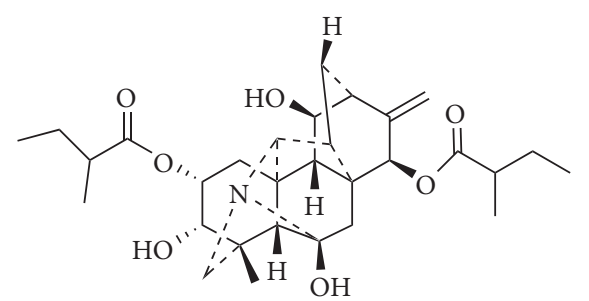

130 Anthriscifolsine C

Figure 6: Continued. 


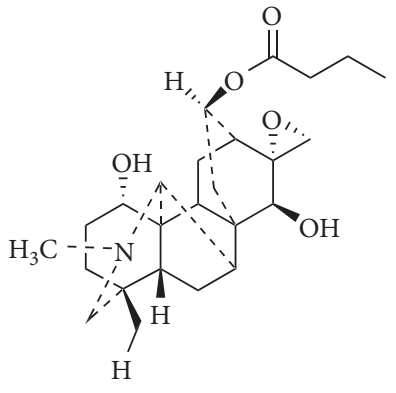

131 Anthriscifolmine A<smiles>CC(C)C(=O)O</smiles>

$\mathrm{HO}$

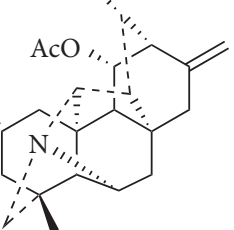

134 Trichodelphinines A

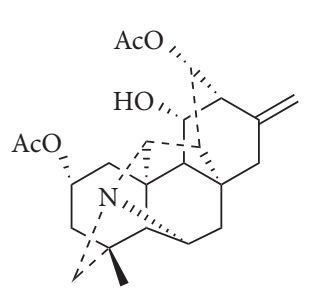

137 Trichodelphinines D

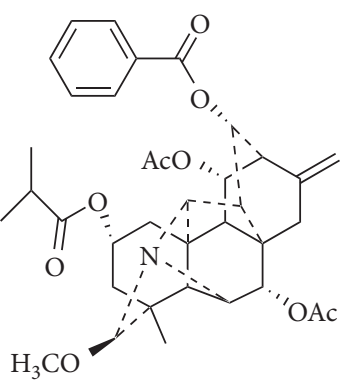

140 Flexiosine

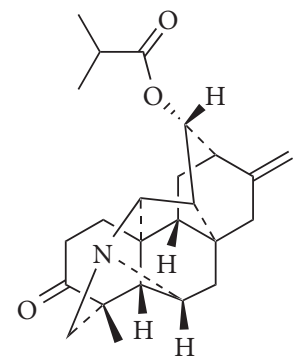

143 Tatsienenseine C

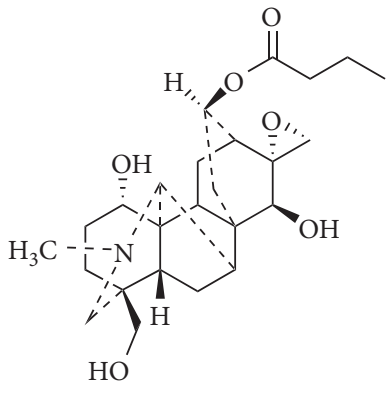

132 Anthriscifolmine B<smiles>CC(C)C(=O)O</smiles>

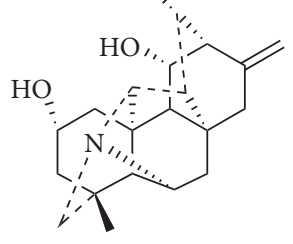

135 Trichodelphinines B

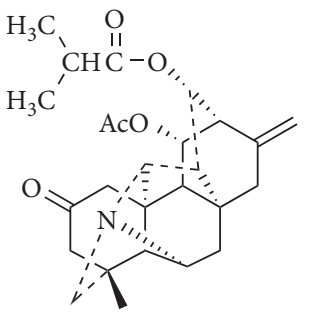

138 Trichodelphinines E

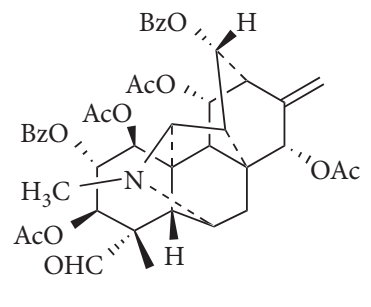

141 Tatsienenseine A

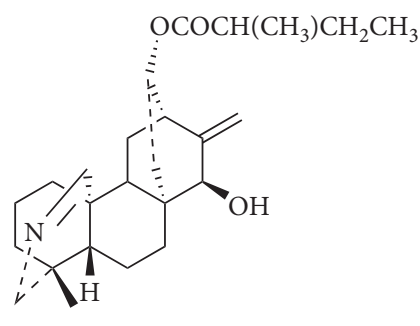

144 13-(2-Methyl butyryl)azitine

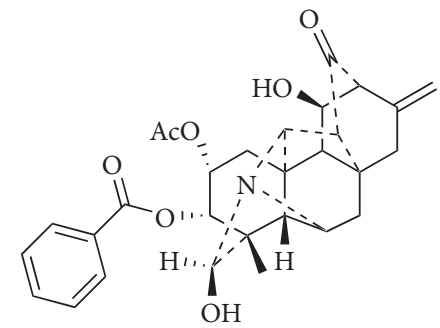

133 Anthriscifolmine C<smiles>CC[C@H](C)C(=O)O</smiles>

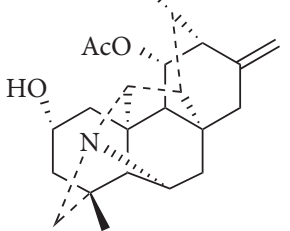

136 Trichodelphinines C

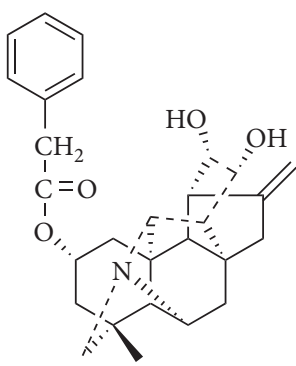

139 Trichodelphinines F

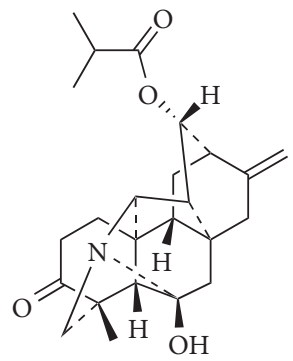

142 Tatsienenseine B

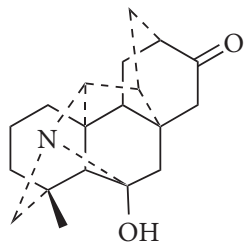

145 Tatsienensine

Figure 6: C-20 diterpenoid alkaloids isolated from Delphinium. 
Table 4: Amide alkaloids isolated from Delphinium.

\begin{tabular}{|c|c|c|c|c|c|}
\hline No. & Compound & Type & Source & $\begin{array}{l}\text { Molecular } \\
\text { formula }\end{array}$ & Reference \\
\hline 146 & $\begin{array}{l}\text { Benzoic acid 2-[(4-methoxy-2-methyl-1,4-dioxobutyl) amino]- } \\
\text { methyl ester }\end{array}$ & Amide & D. grandiflorum Linn & $\mathrm{C}_{14} \mathrm{H}_{17} \mathrm{NO}_{5}$ & {$[21]$} \\
\hline 147 & N-Cinnamoyl-2-phenylethylamine & Amide & D. grandiflorum $\mathrm{L}$. & $\mathrm{C}_{17} \mathrm{H}_{17} \mathrm{NO}$ & {$[48]$} \\
\hline 148 & Delamide A & Amide & D. brunonianum & $\mathrm{C}_{13} \mathrm{H}_{13} \mathrm{NO}_{4}$ & {$[58]$} \\
\hline 149 & Delamide B & Amide & D. brunonianum & $\mathrm{C}_{14} \mathrm{H}_{17} \mathrm{NO}_{6}$ & {$[58]$} \\
\hline 150 & Delamide C & Amide & D. brunonianum & $\mathrm{C}_{13} \mathrm{H}_{15} \mathrm{NO}_{5}$ & {$[58]$} \\
\hline 151 & Delamide D & Amide & D. brunonianum & $\mathrm{C}_{13} \mathrm{H}_{15} \mathrm{NO}_{5}$ & {$[58]$} \\
\hline 152 & Delamide E & Amide & D. brunonianum & $\mathrm{C}_{13} \mathrm{H}_{15} \mathrm{NO}_{5}$ & {$[58]$} \\
\hline 153 & Benzoic,4-[(3,4-dimethoxybenzoyl) amino]-3-hydroxy-methyl ester & Amide & $\begin{array}{l}\text { D. brunonianum } \\
\text { Royle }\end{array}$ & $\mathrm{C}_{17} \mathrm{H}_{17} \mathrm{NO}_{6}$ & {$[59]$} \\
\hline 154 & $\begin{array}{l}\text { Benzoic acid 2-[(4-methoxy-3-methyl-1,4-dioxobutyl) amino]- } \\
\text { methyl ester }\end{array}$ & Amide & $\begin{array}{l}\text { D. brunonianum } \\
\text { Royle }\end{array}$ & $\mathrm{C}_{14} \mathrm{H}_{17} \mathrm{NO}_{5}$ & {$[59]$} \\
\hline
\end{tabular}<smiles>COC(=O)CC(C)C(=O)Nc1ccccc1C(=O)OC</smiles>

146 Benzoic acid 2-[ (4-methoxy-2methyl- 1,4- dioxobutyl) amino]methylester<smiles>COC(=O)c1ccccc1N1C(=O)CC(C)C1=O</smiles>

148 Delamide A<smiles>COC(=O)c1ccccc1NC(=O)CC(C)(O)C(=O)OC</smiles>

149 Delamide B<smiles>O=C(/C=C/c1ccccc1)NCCc1ccccc1</smiles>

147 N-Cinnamoyl-2-phenylethylamine<smiles>COC(=O)C(C)C(=O)Nc1ccccc1C(=O)O</smiles>

151 Delamide D<smiles>COC(=O)c1ccc(NC(=O)c2ccc(OC)c(OC)c2)c(O)c1</smiles>

153 Benzoic,4-[(3,4dimethoxybenzoyl) amino]3-hydroxy-methyl ester<smiles>COC(=O)CC(C)C(=O)Nc1ccccc1C(=O)O</smiles>

150 Delamide C

Figure 7: Amide alkaloids isolated from Delphinium.

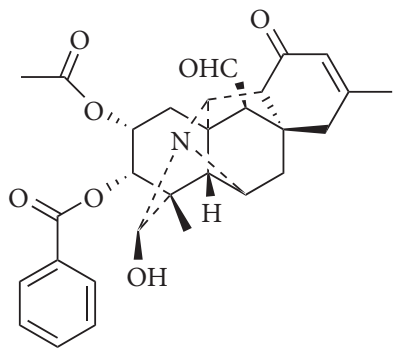

155 Anthriscifolsine A

FIgURE 8: Other alkaloids isolated from Delphinium. 


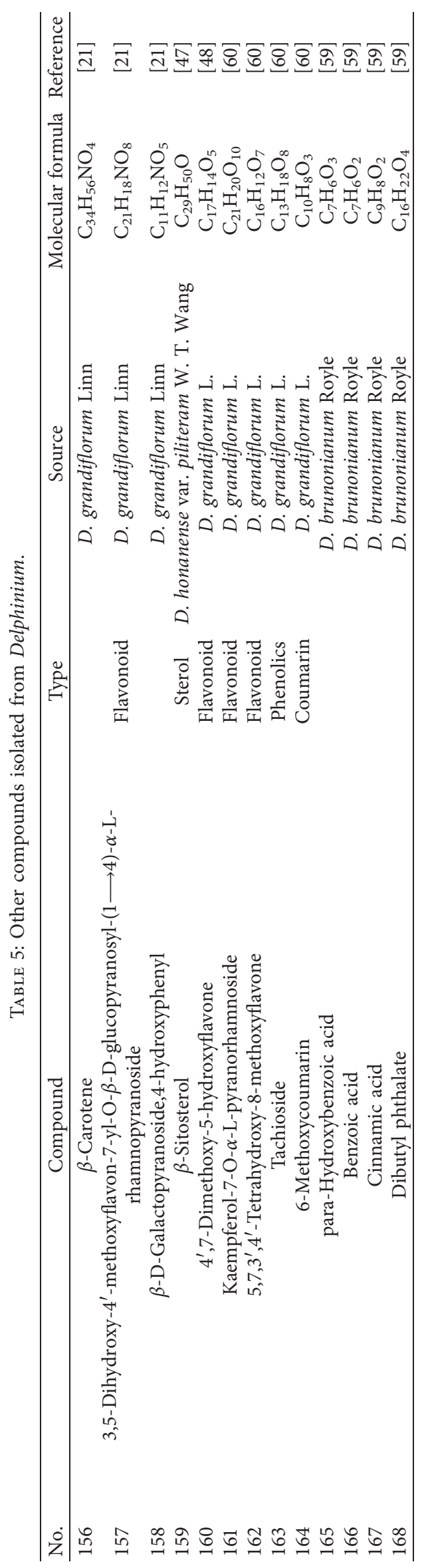


<smiles>CO[C@H]1CC[C@@]2(C)C(=CCC3C4CC[C@H]([C@@H](C)CCC(C)C(C)C)[C@]4(C)CCC32)C1</smiles>

$156 \beta$-Carotene<smiles>COc1ccc(O)cc1</smiles>

$158 \beta$-D-Galactopyranoside, 4-hydroxyphenyl<smiles>[R11]COc1cc(O)c2c(=O)cc(-c3ccc(OC)cc3)oc2c1</smiles>

157 3,5-Dihydroxy-4' -methoxyflavon-7-ylO- $\beta$-D-glucopyranosyl- $(1 \rightarrow 4)-\alpha$-Lrhamnopyranoside<smiles>CC[C@H](CC[C@@H](C)C1CCC2[C@H]3CC=C4C[C@@H](O)CC[C@]4(C)C3CC[C@@]12C)C(C)C</smiles>

$159 \beta$-Sitosterol<smiles>COc1ccc(-c2cc(=O)c3c(O)cc(OC)cc3o2)cc1</smiles>

$1604^{\prime}$, 7-Dimethoxy-5-hydroxyflavone<smiles>C[C@@H]1O[C@H](Oc2cc(O)c3c(=O)c(O)c(-c4ccc(O)cc4)oc3c2)[C@H](O)[C@H](O)[C@@H]1O</smiles>

161 Kaempferol-7-O- $\alpha$-L-pyranorhamnoside

$\mathrm{OH}$<smiles>COc1ccc2oc(=O)ccc2c1</smiles>

163 Tachioside<smiles>O=C(O)c1ccccc1</smiles>

166 Benzoic acid
164 6-Methoxycoumarin<smiles>O=C(O)/C=C/c1ccccc1</smiles>

167 Cinnamic acid<smiles>COc1c(O)cc(O)c2c(=O)cc(-c3ccc(O)c(O)c3)oc12</smiles>

$1625,7,3^{\prime}, 4^{\prime}$-Tetrahydroxy-8-methoxyflavone 
Although there are many research studies, the pharmacological effects on the antibacterial, analgesic, anti-inflammatory, antidepressant, anticancer, antipulmonary fibrosis, antifeedant, and antiparasite effects of Delphinium are mainly on the crude extracts and few on compounds.

\section{Future Prospects}

The genus Delphinium is rich in new and novel compounds, but the current research is only focused on several species. In the future, more new compounds should be investigated from other species in depth. The pharmacological effects of Delphinium are extensive, but the current research is limited to extracts, so it is necessary to focus on the effects of the compounds from Delphinium and the structure-activity relationship in the future.

\section{Data Availability}

The data supporting this article are from previously reported studies, which have been cited. The data are available from the corresponding author upon request.

\section{Conflicts of Interest}

All authors declare that they have no conflicts of interest.

\section{Authors' Contributions}

Sitan Chen and Lijun Meng contributed equally to this work.

\section{Acknowledgments}

This work was supported by the Basic Project in Science and Technology Agency of Kaifeng City (1908007).

\section{References}

[1] W. C. Wang and J. W. Michael, Flora of China, pp. 223-274, Flora of China Editorial Committee of Chinese Academy of Sciences, Beijing, China, 2001.

[2] L. H. Shan, Studies on Diterpenoid Alkaloids Constituents of Four Herb Aconitum, Southwest Jiaotong University, Chengdu, China, 2017.

[3] Flora of China Editorial Committee of Chinese Academy of Sciences, Flora Reipublicae Popularis Sinicae, p. 326, Flora of China Editorial Committee of Chinese Academy of Sciences, Beijing, China, 1979.

[4] F. P. Wang, Q. H. Chen, and X. Y. Liu, "Diterpenoid alkaloids," Natural Product Reports, vol. 27, no. 4, p. 529, 2010.

[5] Teaching and Research Office of Organic Chemistry, Department of Chemistry, Nanjing University, Organic chemistry, p. 329, Higher Education Press, Beijing, China, 1988.

[6] H. X. Kuang, Chemistry of Chinese Materia Medica, China Press of Traditional Chinese Medicine, Beijing, China, 2nd edition, 1998.

[7] F. P. Wang, Alkaloids Chemistrypp. 410-429, Chemical and Industry Press, Beijing, China, 1st edition, 2008.

[8] T. P. Yin, Z. H. Luo, L. Cai, and Z. T. Ding, "Research progress and NMR spectral features of natural $\mathrm{C}_{19}$-diterpenoid alkaloids," Chinese Journal of Magnetic Resonance, vol. 36, no. 01, pp. 113-126, 2019.

[9] Q. Zhang, B. Xu, Q. Jia, and Y. M. Li, “Advances in chemical constituents and pharmacological activities of C18 diterpenoid alkaloids," Chinese Traditional Patent Medicine, vol. 38, no. 5, pp. 1109-1114, 2016.

[10] S. M. Xie, C. Z. Lin, D. W. Zeren et al., "Review of phytochemical and pharmacological researches of medicinal plants from Genus Delphinium of Rannuculaceae," Pharmacy Today, vol. 21, no. 4, pp. 197-201, 2011.

[11] S. J. Wu, Chemical Constituents of Modern Medicinal Herbpp. 936-948, Chinese Medical Science and Technology Press, Beijing, China, 1st edition, 2002.

[12] F.-P. Wang and X.-T. Liang, "C20-diterpenoid alkaloids," The Alkaloids: Chemistry and Biology, vol. 59, pp. 1-280, 2002.

[13] K. B. m. Birnbaum, "X-ray crystal structure of delnudine, a novel alkaloid," Tetrahedron Letters, vol. 10, no. 60, pp. 5245-5246, 1969.

[14] R. Y. Li, F. Feng, and J. H. Liu, "Advance in studies on structure-activity relationships of $\mathrm{C}_{20}$-Diterpenoid alkaloids," Strait Pharmaceutical Journal, vol. 25, no. 12, pp. 1-4, 2013.

[15] Y. Liang, J. L. Wu, X. Li et al., "Anti-cancer and anti-inflammatory new vakognavine-type alkaloid from the roots of Aconitum carmichaelii," Tetrahedron Letters, vol. 57, no. 52, pp. 5881-5884, 2016.

[16] L. S. Ding and Y. Z. Chen, "Natural $C_{20}$ diterpene alkaloids," Natural Product Research and Development, vol. 2, no. 3, pp. 74-88, 1990.

[17] L. Song, X.-X. Liang, D.-L. Chen, X.-X. Jian, and F.-P. Wang, "New C18-Diterpenoid Alkaloids from Delphinium anthriscifolium var. savatieri," Chemical \& Pharmaceutical Bulletin, vol. 55, no. 6, pp. 918-921, 2007.

[18] L. Jian, X.-Y. Liu, and Q.-H. Chen, "New C19- and C18Diterpenoid Alkaloids from Delphinium anthriscifolium var. savatieri," Chemical \& Pharmaceutical Bulletin, vol. 57, no. 2, pp. 158-161, 2009.

[19] W. J. Wang, B. Zhao, J. Y. Zhao et al., "Three new diterpenoid alkaloids from Delphinium naviculare var. lasiocarpum W. T. Wang," Phytochemistry Letters, vol. 33, pp. 12-16, 2019.

[20] S. Wang, X. L. Zhou, X. M. Gong et al., "Norditerpenoid alkaloids from Delphinium anthriscifolium," Journal of Asian Natural Products Research, vol. 18, no. 2, pp. 1-6, 2015.

[21] Z. D. Nan, Studies on the Chemical Constituents of Delphinium grandifloru linn, Lanzhou University, Lanzhou, China, 2010.

[22] Z. D. Nan, H. Z. Ren, X. A. Li et al., "Separation and identification of diterpenoid alkaloids from Delphinium grandiflorum," Chinese Journal of Experimental Traditional Medical Formulae, vol. 23, no. 24, pp. 71-77, 2017.

[23] U. Kolak, M. Oeztuerk, F. Oezgoekce et al., "Norditerpene alkaloids from Delphinium linearilobum and antioxidant activity," Phytochemistry, vol. 67, no. 19, pp. 2170-2175, 2006.

[24] L. H. Shan, J. F. Zhang, L. Chen et al., "Two new C18diterpenoid alkaloids from Delphinium anthriscifolium," Natural Product Communications, vol. 10, no. 12, pp. 2067-2068, 2015.

[25] L.-H. Shan, J.-F. Zhang, F. Gao, S. Huang, and X.-L. Zhou, "C18-Diterpenoid alkaloids from Delphinium anthriscifolium var. majus," Journal of Asian Natural Products Research, vol. 20, no. 5, pp. 423-430, 2018.

[26] K. Huang, E. Asakawa, Y. Tosho et al., "Four new diterpenoid alkaloids from Delphinium elatum," Phytochemistry Letters, vol. 17, pp. 190-193, 2016. 
[27] K. Nakata, R. Chiba, R. Kanazawa et al., "Six new norditerpenoid alkaloids from Delphinium elatum," Phytochemistry Letters, vol. 12, pp. 79-83, 2015.

[28] F. Z. Matsuoka, Q. H. Chen, and F. P. Wang, "Diterpenoid alkaloids from Delphinium yunnanense," Helvetica Chimica Acta, vol. 94, no. 2, pp. 254-260, 2011.

[29] J.-F. Zhang, R.-Y. Dai, L.-H. Shan et al., "Iliensines A and B: two new C 19 -diterpenoid alkaloids from Delphinium iliense," Phytochemistry Letters, vol. 17, pp. 299-303, 2016.

[30] W. J. Xu, B. Zhao, Z. Ruzi et al., "Norditerpenoid alkaloids from Delphinium pseudoaemulans C. Y. Yang et B. Wang," Phytochemistry, vol. 156, pp. 234-240, 2018.

[31] L. Shan, L. Chen, and F. Gao, "Diterpenoid alkaloids from Delphinium naviculare var. lasiocarpum with their antifeedant activity on Spodoptera exigua," Natural Product Research, vol. 33, no. 22, pp. 3254-3259, 2018.

[32] D. Y. Zhou, H. A. Aisa, S. K. Usmanova, and "Shawurensine, "Shawurensine, a new C19-diterpenoid alkaloid from Delphinium shawurense," Chemistry of Natural Compounds, vol. 43, no. 3, pp. 298-301, 2007.

[33] B. Zhao, S. K. Usmanova, A. Yili, A. Kawuli, R. Abdulla, and H. A. Aisa, "New C19-norditerpenoid alkaloid from Delphinium shawurense," Chemistry of Natural Compounds, vol. 51, no. 3, pp. 519-522, 2015.

[34] L. Kawuli, Y. B. Zhang, L. Zhuang et al., "Diterpenoid alkaloids from Delphinium ajacis and their anti-RSV activities," Planta Medica, vol. 83, no. 1-2, pp. 111-116, 2016.

[35] C.-Z. Wang, Z.-J. Liu, and Z.-D. Bairi, "A new diterpenoid alkaloid isolated from Delphinium caeruleum," Chinese Journal of Natural Medicines, vol. 15, no. 1, pp. 45-48, 2017.

[36] N.-H. Zhu, Y.-B. Zhang, W. Li et al., "Grandiflodines A and B, two novel diterpenoid alkaloids from Delphinium grandiflorum," RSC Advances, vol. 7, no. 39, pp. 24129-24132, 2017.

[37] F.-Z. Li, D.-L. Chen, and Q.-H. Chen, "Diterpenoid alkaloids from Delphinium majus," Journal of Natural Products, vol. 72, no. 1, pp. 18-23, 2009.

[38] X. X. Wang, D. L. Chen, and F. P. Wang, "Two new C19diterpenoid alkaloids from Delphinium davidii Franch," Chinese Chemical Letters, vol. 17, no. 11, pp. 1473-1476, 2007.

[39] P. Tang, D. L. Chen, Q. H. Chen et al., "Three new C19diterpenoid alkaloids from Delphinium laxicymosum var. pilostachyum," Chinese Chemical Letters, vol. 18, no. 6, pp. 700-703, 2007.

[40] J. Li, D.-L. Chen, and X.-X. Jian, "New diterpenoid alkaloids from the roots of Delphinium tiantaishanense," Molecules, vol. 12, no. 3, pp. 353-360, 2007.

[41] X. L. Wang, Q. H. Chen, and F. P. Wang, "New C19-diterpenoid alkaloids from Delphinium trifoliolatum," Chemical and Pharmaceutical Bulletin, vol. 52, no. 4, pp. 381-383, 2004.

[42] G. D. Jess, G. R. Juan, and H. Werner, "Alkaloids from Delphinium pentagynum," Phytochemistry, vol. 65, no. 14, pp. 2123-2127, 2004.

[43] X.-L. Zhou, Q.-H. Chen, and F.-P. Wang, "Three new C19diterpenoid alkaloids from Delphinium giraldii," Chemical \& Pharmaceutical Bulletin, vol. 52, no. 4, pp. 456-458, 2004.

[44] X. L. Zhou, Q. H. Chen, and F. P. Wang, "Three new lycoctonine-type C19-diterpenoid alkaloids from Delphinium giraldii," Heterocycles, vol. 63, no. 1, pp. 123-128, 2004.

[45] L. P. Yan, D. L. Chen, and F. P. Wang, "Structure elucidation of diterpenoid alkaloids from Delphinium campylocentrum," Organic Chemistry, vol. 27, no. 8, pp. 976-980, 2007.

[46] X. X. Liang, Studies on the Chemical Constituents of Alkaloids in Delphinium davidii, Sichuan University, Chengdu, China, 2007.
[47] Y. Q. He, Z. Y. Ma, Q. Yang et al., "Study on chemical constituents from Delphinium honanense var.piliteram," China Journal of Chinese Materia Medica, vol. 33, no. 23, p. 2784, 2008.

[48] Y. L. Han, Studies on Chemical Constitudents of Gueldenstaedita multiflora Bge. and Delphinium grandiflorum L, Northwest Normal University, Lanzhou, China, 2007.

[49] L. H. Shan, L. Chen, F. Gao et al., "Diterpenoid alkaloids from Delphinium anthriscifolium var. majus," Scientific Reports, vol. 7, no. 1, 2017.

[50] J. F. Zhang, L. H. Shan, F. Gao et al., "Five new $\mathrm{C}_{19}$-diterpenoid alkaloids from Delphinium tianshanicum W. T. wang," Chemistry and Biodiversity, vol. 14, no. 4, Article ID e1600297, 2017.

[51] H. Yamashita, M. Katoh, A. Kokubun et al., "new $\mathrm{C}_{19}$ -diterpenoid alkaloids from Delphinium elatum," Phytochemistry Letters, vol. 24, pp. 6-9, 2018.

[52] X.-Y. Uchimura, Q.-H. Chen, and F.-P. Wang, "New C20Diterpenoid Alkaloids from Delphinium anthriscifolium var. savatieri," Helvetica Chimica Acta, vol. 92, no. 4, pp. 745-752, 2009.

[53] C. Z. Lin, Z. X. Zhao, S. M. Xie et al., "Diterpenoid alkaloids and flavonoids from Delphinium trichophorum," Phytochemistry, vol. 97, pp. 88-95, 2014.

[54] T. M. Gabbasov, E. M. Tsyrlina, D. M. Anatov, and M. S. Yunusov, "Flexiosine, a new C20-diterpene alkaloid from roots of Delphinium flexuosum," Chemistry of Natural Compounds, vol. 53, no. 1, pp. 105-108, 2017.

[55] F.-Z. Spirikhin, Q.-H. Chen, and X.-Y. Liu, "Diterpenoid alkaloids from Delphinium tatsienense," Helvetica Chimica Acta, vol. 94, no. 5, pp. 853-858, 2011.

[56] M. S. Wang and K. Alfred, "Diterpenoid alkaloids from the roots of Delphinium scabriflorum," Journal of Natural Products, vol. 67, no. 9, pp. 1574-1576, 2004.

[57] F. Z. Chen, S. H. Li, Q. Zhao, and X. J Gou, "Tatsienensine, a norditerpenoid alkaloid from Delphinium tatsienense," Natural Product Communications, vol. 14, no. 11, pp. 1-3, 2019.

[58] Y.-S. Zou, Z. Dawa, C.-Z. Lin et al., "New amide alkaloids from Delphinium brunonianum," Fitoterapia, vol. 136, Article ID 104186, 2019.

[59] Z. D. Zhang, X. A. Li, Y. X. Chen et al., "Chemical constituents from the Delphinium grandiflorum herbs," Journal of Chinese Medicinal Materials, vol. 40, no. 9, pp. 2077-2080, 2017.

[60] Y. S. Zou, D. W. Zeren, C. Z. Lin et al., "Chemical constituents from Delphinium brunonianum," Journal of Chinese Medicinal Materials, vol. 42, no. 8, pp. 1806-1809, 2019.

[61] P. T. Hari, P. S. Ram, P. T. Yagna et al., "An assessment of ethnomedicinal use, chemical constituents analysis and bioactivity evaluation on high altitude medicinal plant Delphinium brunonianum of Manang District," Nepal Journal of Science and Technology, vol. 12, pp. 111-118, 2011.

[62] S. Y. Ren, G. L. Qiansiri, H. Y. Wang et al., "Extraction and antibacterial test of alkaloid from root of Delphinium grandiflorum," Journal of Dalian Polytechnic University, vol. 28, no. 4, pp. 248-250, 2009.

[63] I. Zaheer, S. Z. Rahman, R. A. Khan et al., "Evaluation of analgesic activity of extracts of Delphinium denudatum in animal models: a dose dependent pre-clinical trial (Article)," Journal of Clinical and Diagnostic Research, vol. 12, no. 12, pp. FC1-FC4, 2018.

[64] Y. V. Nesterova, T. N. Povetieva, Y. G. Nagornyak, T. V. Vetoshkina, and T. I. Andreeva, "Correction of adjuvant arthritis with delphinium extracts and alkaloids," Bulletin of 
Experimental Biology and Medicine, vol. 147, no. 6, pp. 711$714,2009$.

[65] G. S. Suslov, Z. P. Jia, S. Z. Qiao et al., "Experimental studies on haemostasis and analgesic effect of Delphinium grandiflorum L. var. leiocarpum," Chinese Journal of Hospital Pharmacy, vol. 30, no. 11, pp. 898-900, 2010.

[66] Y. V. Nesterova, T. N. Poveteva, Y. G. Nagornyak, T. I. Andreeva, and N. I. Suslov, "Effects of bioactive substances from tall delphinium on the development of acute inflammation of different genesis," Bulletin of Experimental Biology and Medicine, vol. 145, no. 6, pp. 724-727, 2008.

[67] L. Andreeva and Y. J. Liu, "Study of Delphinium grandiflorum flavonoids on inhibition of inflammation in mice," China Modern Medcine, vol. 18, no. 32, p. 14+59, 2011.

[68] M. A. Ebrahimzadeh, S. F. Nabavi, S. M. Nabavi et al., "Biological and pharmacological effects of Delphinium elbursense," African Journal of Biotechnology, vol. 9, no. 34, pp. 5548-5555, 2010.

[69] A. Mohammad, G. A. Kumar, and A. K. Najam, "In vivo psychopharmacological investigation of Delphinium denudatum and Amaranthus spinosus extracts on Wistar rats," Basic and Clinical Neuroscience Journal, vol. 8, no. 6, pp. 503-512, 2017.

[70] S. J. Zheng, C. Xu, J. Yang et al., "In vitro anticancer screening of Tibetan medicines," Journal of Huazhong Normal University (Natural Sciences), vol. 51, no. 3, pp. 328-334, 2017.

[71] C. Z. Lin, C. C. Zhu, F. L. Liu et al., "The protective effects of extracts of Delphinium trichophorum on bleomycin-induced pulmonary fibrosis in rats," Journal of Chinese Medicinal Materials, vol. 41, no. 5, pp. 1181-1185, 2018.

[72] C. González and G. Guadaño, "Antifeedant delphinium diterpenoid alkaloids. Structure-activity relationships," Journal of Agricultural and Food Chemistry, vol. 46, no. 1, 1998.

[73] M. Reina, R. Mancha, A. Gonzalez-Coloma, M. Bailen, M. L. Rodriguez, and R. A. Martinez-Diaz, "Diterpenoid alkaloids from Delphinium gracile," Natural Product Research, vol. 21, no. 12, pp. 1048-1055, 2007. 